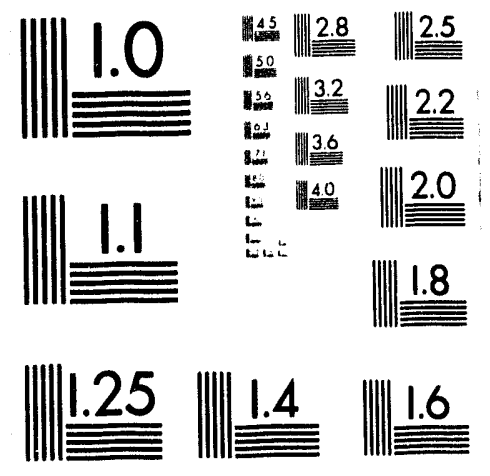



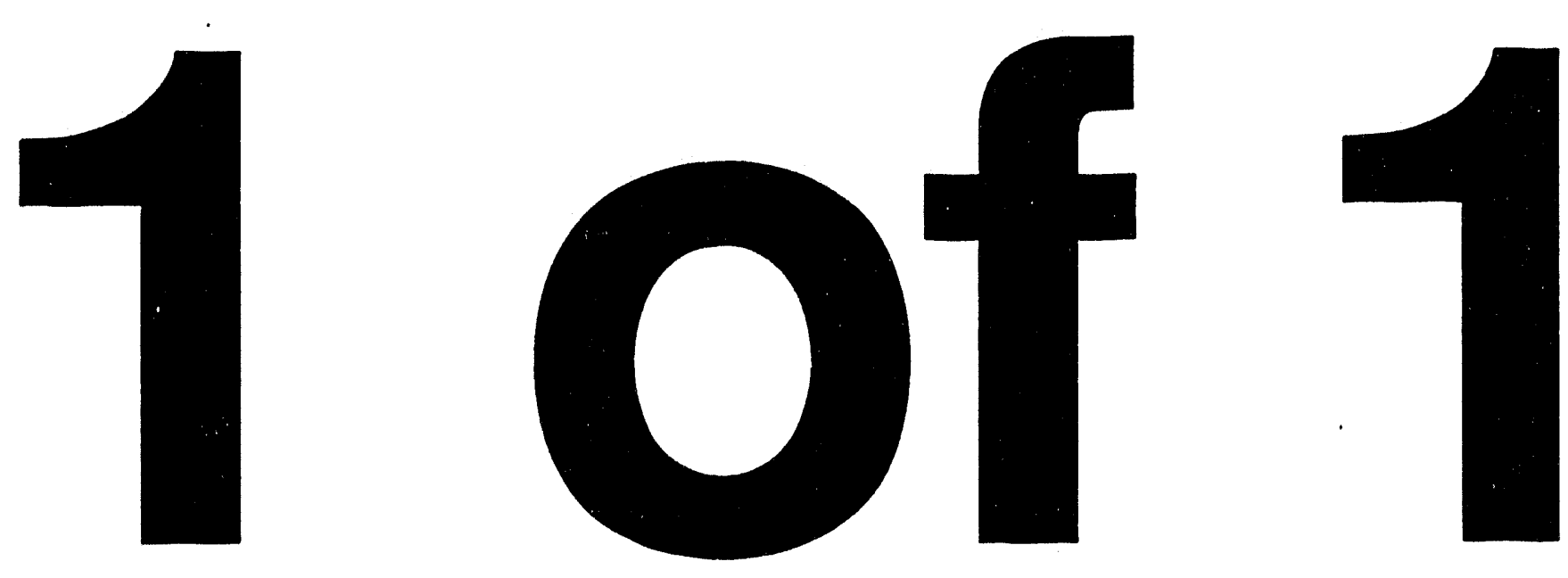
UCRL-ID 112771

$$
\begin{array}{r}
\text { OSTI } \\
\text { nOV } 191933
\end{array}
$$

Reprinted from Engineering Research, Development, and Technology.

Thrust Area Report FY 92

UCRL 53868-92

\section{Computational Electronics and Electromagnetics}

\section{John F. DeFord}

March, 1993

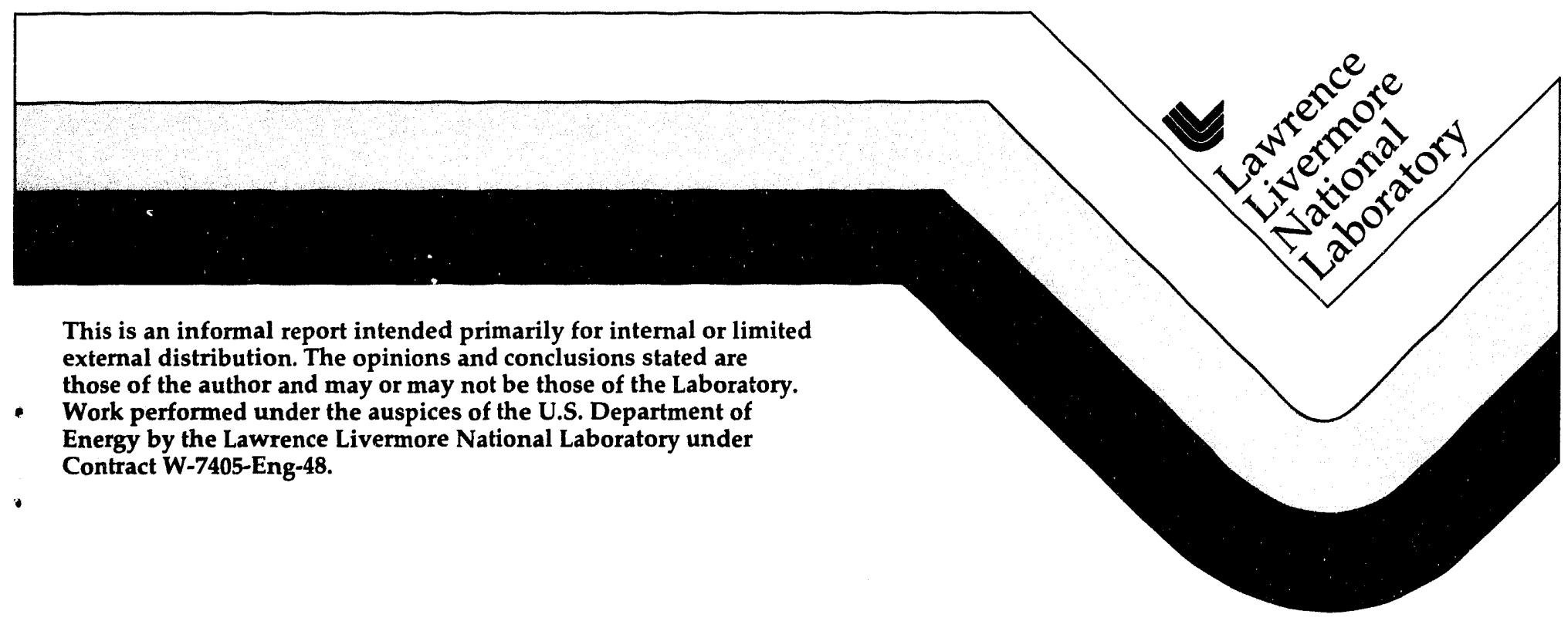


Disclaimer

This document was prepared as an account of work sponsored by an agency of the United States Government. Neither the United States Government nor the University of California nor any of their employees, makes any warranty, express or implied, or assumes any legal liability or responsibility for the accuracy, completeness, or usefulness of any information, apparatus, product, or process disclosed, or represents that its use would not infringe privately owned rights. Reference herein to any specific commercial products, process, or service by trade name, trademark, manufacturer, or otherwise, does not necessarily constitute or imply its endorsement, recommendation, or favoring by the United States Government or the University of California. The views and opinions of authors expressed herein do not necessarily state or reflect those of the United States Government or the University of California and shall not be used for advertising or product endorsement purposes.

This report has been reproduced

directly from the best available copy.

Available to DOE and DOE contractors from the Office of Scientific and Technical Information P.O. Box 62, Oak Ridge, TN 37831

Prices available from (615) 576-8401, FTS 626-8401

Available to the public from the National Technical Information Service

U.S. Department of Commerce 5285 Port Royal Rd., Springfield, VA 22161 


\title{
Computational Electronics and Electromagnetics
}

\author{
John F. DeFord
}




\section{Computational Electronics and Electromagnetics}

The Computational Electronics and Electromagnetics thrust area is a focal point for computer modeling activities in electronics and electromagnetics in the Electronics Engineering Department of Lawrence Livermore National Laboratory (LLNL). Traditionally, we have focused our 12 efforts in technical areas of importance to existing and developing LLNL programs, and this continues to form the basis for much of our research. A relatively new and increasingly important emphasis for the thrust area is the formation of partnerships with industry and the application of our simulation technology and expertise to the solution of problems faced by industry.

The activities of the thrust area fall into three broad categories: (1) the development of theoretical and computational models of electronic and electromagnetic phenomena, (2) the development of useful and robust software tools based on these models, and (3) the application of these tools to programmatic and industrial problems. In FY-92, we worked on projects in all of the areas outlined above. The object of our work on numerical electromagnetic algorithms continues to be the improvement of time-domain algorithms for electromagnetic simulation on unstructured conforming grids. The thrust area is also investigating various technologies for conforming-grid mesh generation to simplify the application of our advanced field solvers to design proble: ns involving complicated geometries. We are developirig a major code suite based on the three-dimensional (3-D), conforming-grid, time-domain code DSI3D. We continue to maintain and distribute the 3-D, finite-difference timedomain (FDTD) code TSAR, which is installed at several dozen university, government, and industry sites. Also, during this past year we have begun to distribute our two-dimensional FDTD accelerator modeling code AMOS, and it is presently being used at several universities and Department of Energy accelerator laboratories. Our principal applications during FY-92 were accelerator components, microwave tubes, photonics, and the evaluation of electromagnetic interference effects in commercial aircraft.

Included in this report are several articles that discuss some of our activities in more detail. The topical areas covered in these articles include computational integrated photonics, the application of massively parallel computers to time-domain modeling, analysis of pulse propagation through concrete for bridge inspection, accelerator component modeling, and the development of tools for semiconductor bandgap calculations.

John F. DeFord Thrust Area Lender 


\section{Section 1}

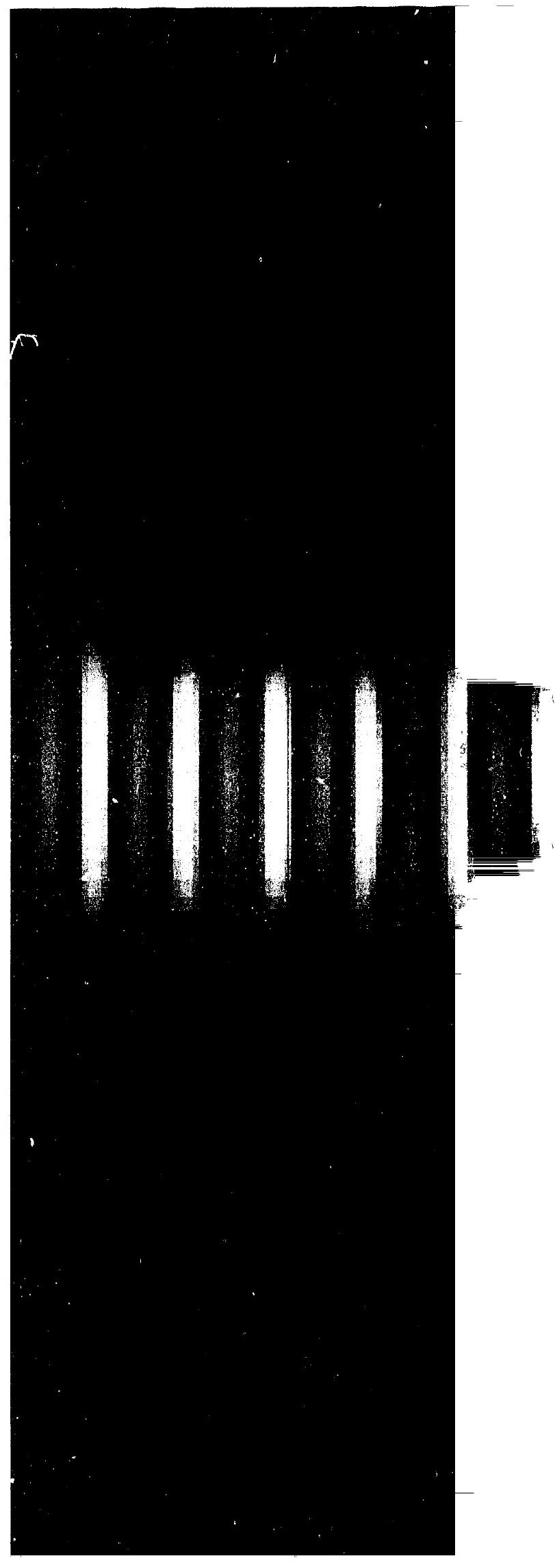




\section{Computational Electronics and Electromagnetics}

Overview

John F. DeFord, Thrust Area Leader

Parallel Computers and Three-Dimensional Computational Electromagnetics

Niel K. Madsen

Computational Integrated Photonics

Raymond J. Hawkins, Jeffery S. Kallman, and Richard W. Ziolkowski

Analysis of High-Average-Power, Millimeter-Wave Microwave Components and Induction Linear Accelerator Modules

Clifford C. Shang, John F. DeFord, and Malcolm Caplan $1-13$

Electromagnetic Modeling and Experiments for Dispersive Media Scott D. Nelson and Carlos A. Avalle $1-21$

Band Gap Engineering for Infrared Detectors

J. Brian Grant 


\title{
Parallel Computers and Three- Dimensional Computational Electromagnetics
}

\author{
Niel K. Madsen \\ Engineering Research Division \\ Electronics Engineering
}

We have continued to make progress in our ability to use massively parallel processing (MPP) computers to solve large, computational, electromagnetics problems. In FY-92, our primary emphasis has been to produce a message-passing version of the preprocessor, PREDSI3D. In addition, the execution module DSI3D has been ported to other parallel machines: the BBN Butterfly, the Thinking Machines CM-5, and the Kendall Square KSR-1 machine. Our DSI3D algorithm and code, together with the ever more capable MPP computers, give us a unique opportunity for significant new contributions to three-dimensional electromagnetic modeling. Two recent applications of DSI3D are presented: (1) full-wave analyses of very-high-frequency optical signals propagating in a weakly guided optical fiber cable; and (2) study of the behavior of whispering-gallery-mode microdisk lasers.

\section{Introduction}

The solution of physical problems whose behavior is governed by Maxwell's equations has been of considerable interest for many years. The propagation of electromagnetic (EM) signals, such as microwaves for communication or radar pulses for the detection of aircraft, are two examples of such problems that have been studied over long periods of time. More recently, other areas such as the design of integrated photonics devices; the design and analysis of electronic interconnects for integrated circuits; and the full-wave analysis of microdisk or thumbtack lasers have been studied by numerically solving Maxwell's equations.

The computational tasks for accurately modeling three-dimensional (3-D) problems that are electromagnetically large are very challenging. Two limitations that have been real impediments to the successful solution for these problems are (1) the lack of good, numerical EM algorithms for dealing with problems with complicated, irregular, and nonorthogonal geometries; and (2) the speed and capacity of even the largest and fastest supercomputers.

Present-day supercomputers, such as the CrayYMP, limit full-wave finite difference or finite vol- ume Maxwell's solutions to computational volumes smaller than about $10^{4} \lambda^{3}$, where $\lambda$ is the wavelength of the EM radiation of interest. For a radar cross-section (RCS) calculation, this limits one to the analysis of scattering from only a small portion of an aircraft fuselage at the upper end of the low-frequency regime, thus neglecting the intra-structure coupling effects that can be important under some conditions. The calculation of the RCS of a complete aircraft, which may be of size $100 \lambda$ in each of three dimensions, may require as many as $10^{9}$ grid or mesh elements. Problems of this extremely large size clearly will require computers with capabilities that are far beyond those of current supercomputers.

New massively parallel processing (MPP) computers have emerged as the most attractive approach for increasing our computing capabilities to the levels required by large, 3-D, EM simulations. Though still evolving rapidly and not as yet completely viable as production computers, they have demonstrated computational speeds that can no longer be ignored.

With their very distributed nature (memory and CPU's) and lack of sophisticated software development tools, MPP computers present new computing challenges in and of themselves. Large 


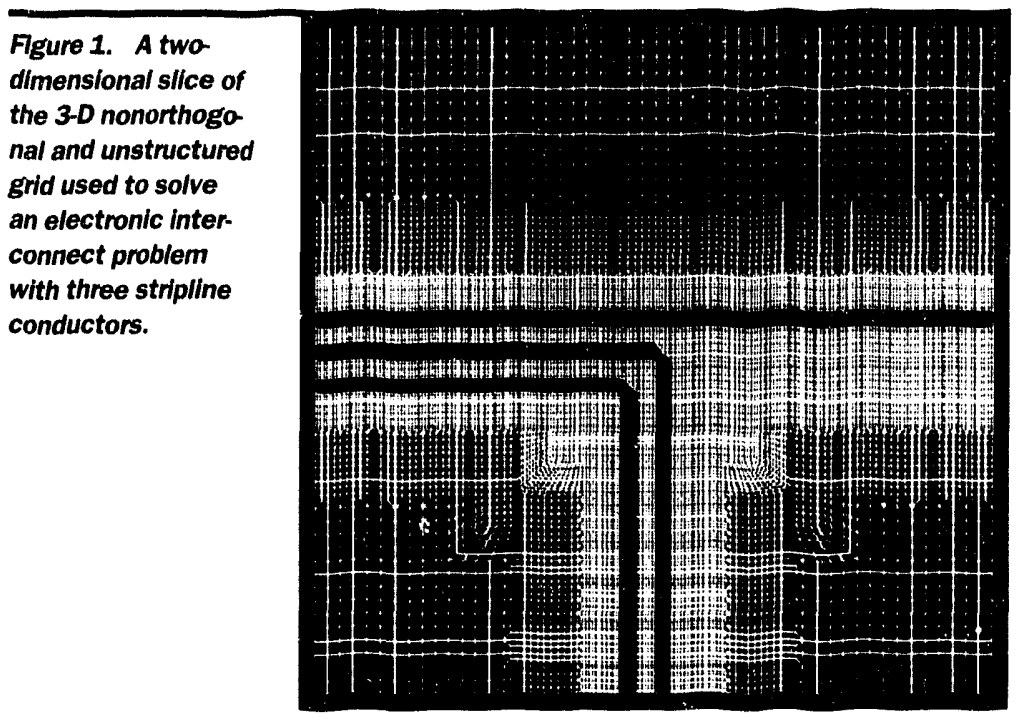

Figure 2. A two dimensional slice showing the automatic partitioning produced by the re cursive spectral bfsectlon method for partitioning the electronic Interconnect problem.

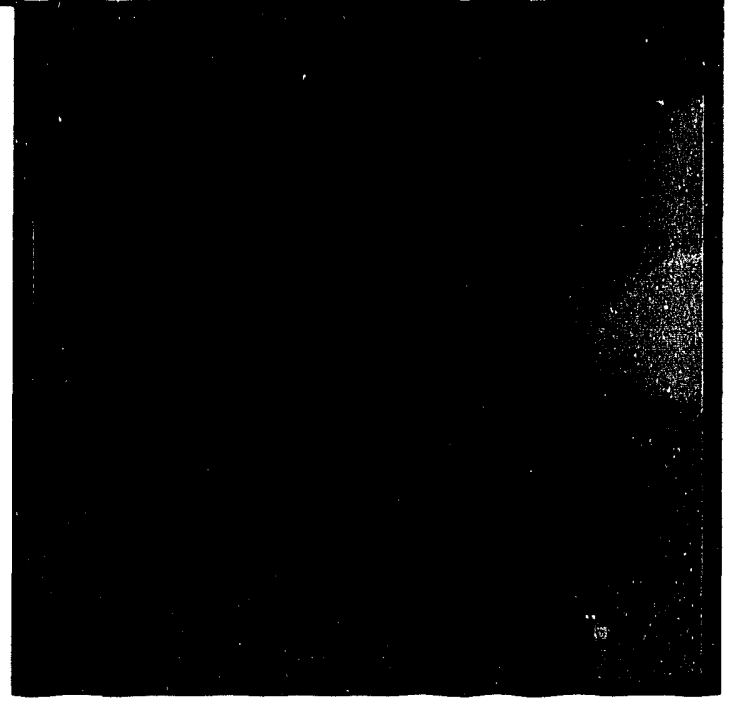

simulation problems must be broken intc, smaller subpieces that can be handled by the individual processors. As a result of this decomposition, data must be efficiently communicated among the processors as required by the numerical algorithm. In the next section, we will describe a new technique that can automatically decompose a problem into smaller subpieces, and also seems to be effective at minimizing the amount of required communication. We will also discuss the performance of our new parallel EM software on one of these newer parallel computers.

Finally, we will show some sample results for two optical applications problems and conclude by indicating our future development directions and thoughts.

\section{Progress}

With our development over the past several years of the new discrete surface integra! (i)SI) methods, the first of the two modeling limitations listed above has been completely overcome, i.e., our new algorithm (implemented in the code DSI3D) has proven to be robust, reliable, and accurate in solving EM problems with complicated and irregular geometries.

The second limitation has been the primary subject of our work for the past two years. Previously, we produced and tested a parallel version of the DSI3D execution module that performs quite efficiently on distributed-memory parallel computers such as the Intel iPSC/860.

\section{Parallel Computation Issues}

Recognizing the ultimate physics limitations of trying to speed up traditional serial processing computers, computer manufacturers have begun to design and build MPP computers with hundreds and even thousands of independent processors. These processors are capable of performing hundreds or thousands of arithmetic computations at the same time.

Typically, these MPP computers are distributed-memory computers, i.e., they have very large total amounts of memory, but each processor has rapid direct access to only a small subset of the total memory. For a processor to obtain access to data not residing in its own memory, some form of communication or message passing among processors is required. This distribution of memory presents new complexities when one desires to solve very large problems. Ultimately, the computer's operating system, compilers, and other software tools will automatically take care of these additional complexities. At present, however, all of these software tools are in a state of infancy, and so solving very large EM problems remains a challenging task.

The efficient partitioning or distribution of the computational tasks and data across the computer's memory and processors is an area of high interest. A good partitioning of a problem among multiple processors should satisfy at least two criteria: (1) the partitioning of a problem should produce subpieces of approximately equal size; and (2) the boundaries between the pieces should be as small as possible. The first requirement is 
imposed to make sure that each processor has about the same amount of computational work to perform; the second requirement is set to try to minimize the amount of inter-processor data communication.

For problems with a regular structure, it is often the case that an efficient partitioning of the problem is visually obvious. However, for unstructured grids with little predictable structure, a good partitioning is rarely obvious and can present a formidable problem. Last year, we reported our initial experience with a very promising new approach. Others ${ }^{2}$ have recently developed a 'recursive spectral bisection' method that seems to meet both of the above criteria. It is based upon the construction of the Laplacian matrix of the dependency graph of the algorithm being used. A dependency graph is produced by linking together variables that depend upon each other, through the underlying solution algorithm (in our case the DSI3Dalgorithm). The partitioning is accomplished by finding the second eigenvalue of the Laplacian matrix and an associated eigenvector, which is referred to as the 'Fiedler vector.' The median value of the entries of the Fiedler vector is computed, and variables that are associated with Fiedler vector entries that are greater than the median value form one piece of the partition, and those less than the median value form the other partition piece. This process can then be applied recursively to partition the entire problem into the desired number of pieces, which must be an integral power of 2.

We have further tested this new, recursive, spectral bisection technique and have found it to be quite effective at meeting the desired criteria for a good partitioning, even for very large problems. Figure 1 shows a two-dimensional cross-section of a 3-D, unstructured interconnect grid; Fig. 2 shows the automatically derived partitioning of this grid cross section into 16 colored subpieces (shades of gray in this rendition).

In addition to the partitioning of an EM problem for MPP solution, there is also the difficulty of producing a version of the unstructured grid code, DSI3D, that runs efficiently on a MPP computer. The primary challenge is to design and implement the passing of data among processors, so that it consumes a small amount of time compared with the time required for computing the solution components. The DSI3D code is really separated into two subpieces: a preprocessing piece, PREDSI3D, which takes the primary grid and the DSI algorithm and derives a dependency graph and update coefficients; and an execution piece, DSI3D,

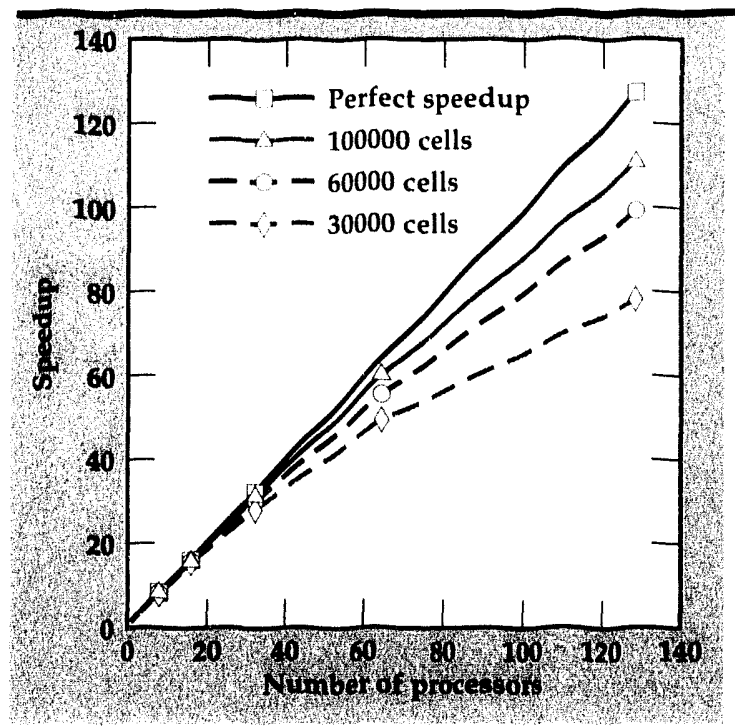

Fgure 3. Parallel performance of DSI3D for waveguide problems of three dif. ferent sizes, using various numbers of processors on the Intel IPSC/860.

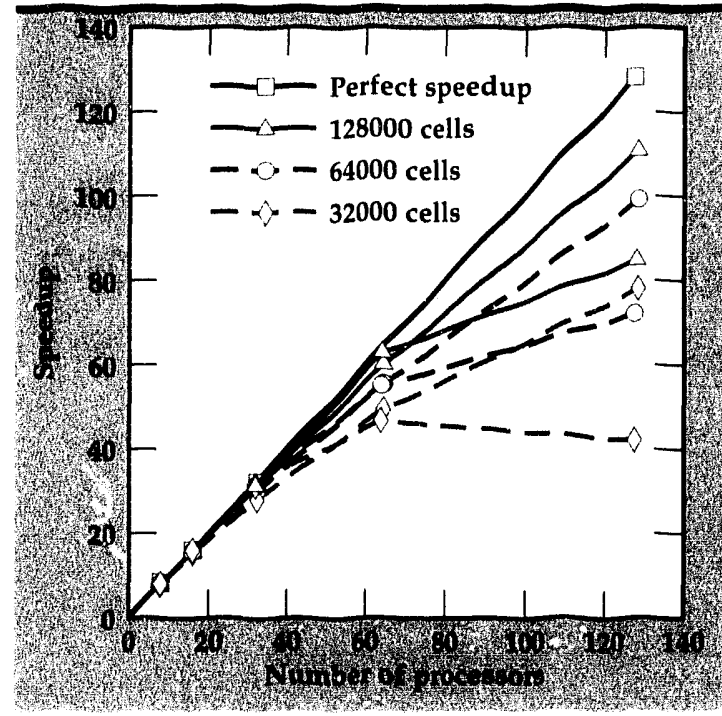

Figure 4. Paralle performance of PREDSI3D for wavegulde problems of three different sizes, using various numbers of processors on the Intel IPSC $/ 860$. which repeatedly uses the dependency graph and coefficients to update the field components in a time-marching manner.

In FY-91, we completed the implementation of message-passing versions of DSI3D for the Intel iPSC / 860 parallel computer. In FY-92, our primary emphasis has been to produce a message-passing version of the preprocessor, PREDSI3D. In addition, the execution module DSI3D has been ported to other parallel machines: the BBN Butterfly, the Thinking Machines CM-5, and the Kendall Square KSR-1 machine. Generally, we have found that if the problem is sufficiently large, there is considerable benefit to using parallel computers. For smaller problems, it is more efficient to use conventional serial-processing computers. Figure 3 shows the performance of DSI3D for waveguide propagation problems of three different sizes, using varying numbers of processors on the Intel iPSC /860 parallel computer. Figure 4 shows the 


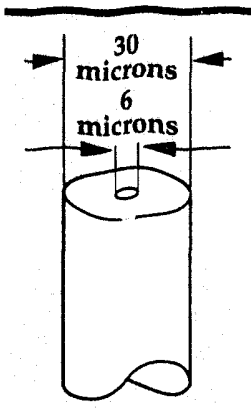

Figure 5. Schematic for a weakly guided fiber optical cable with an offset endcleave. performance of PREDSI3D for a similar set of problems. It is clear from these figures that larger problems run uniformly more efficiently than do smaller problems. The preprocessor, PREDSI3D, in general runs less efficiently than the execution module, becausc it requires considerably more interprocessor communication. However, it is run only once for a particular problem, whereas the execution module may be run repeatedly for the same problem.

\section{Selected Applications}

The overall purpose of our work using MPP computers is to be able to easily solve problems that heretofore have not been solvable on conventional serial-processing computers. While the existing MPP computers are not quite at that level of capability, the next generation will be, and we are now ready to address this type of problem.

One area of new interest to us has been the use of DSI3D to perform full-wave analyses of very-

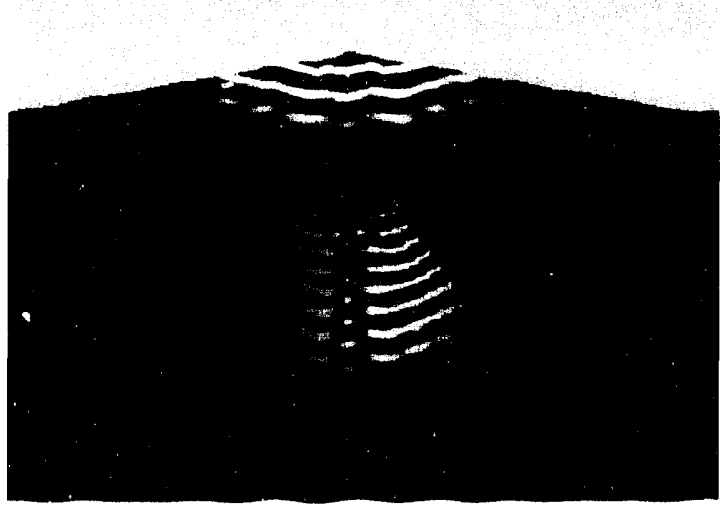

Figure 6. Two-dimensional planar cut in the center of a ffber optical cable with a centered end-cleave, showing the reflected pulse field fringes.

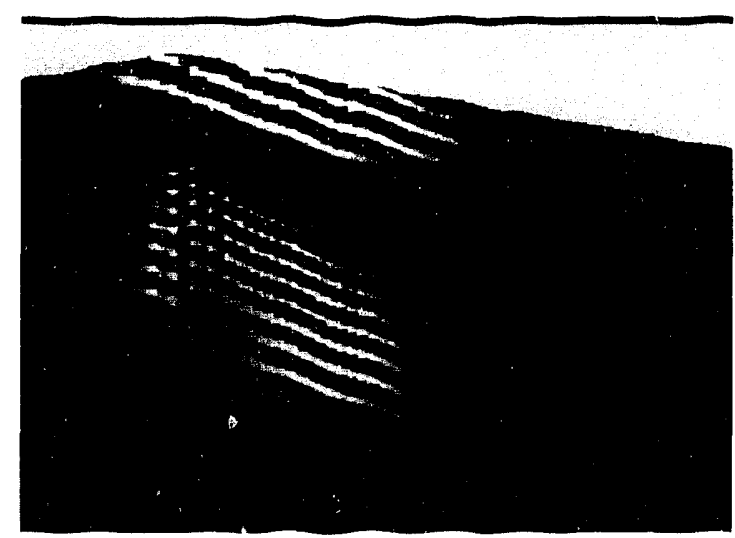

Figure 7. Two-dimensional planar cut in the center of a fiber optical cable with an offset end-cleave, showing the reflected pulse field fringes.

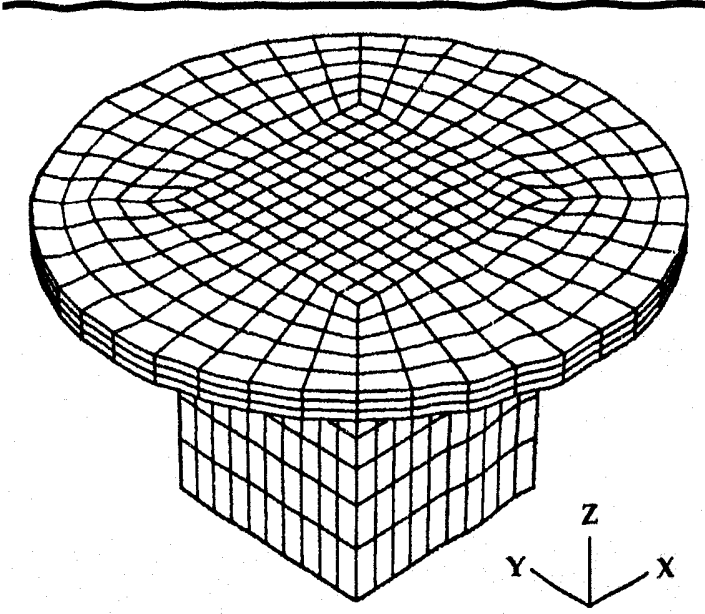

Figure 8. DSI3D grid used to model the behavior of the $\mathrm{mi}$ crodisk laser and pedestal.

high-frequency optical signals propagating in weakly guided optical fiber cables. In splicing optical fibers, it is desired to cleave (or cut) them in a manner so that the cleave is tent-shaped and centered with respect to the fiber core (see Fig. 5). Due to their small size, it is not always easy to determine if the cleave is appropriately centered. One idea for determining if the proper centering exists is to launch optical signals in the fiber cable toward the cleave, and then to analyze the signal reflected from the cleaved end back down the cable. If the cleave is centered, most of the reflected energy should remain in the cable core in the fundamental mode. If the cleave is offset significantly, much of the reflected energy will be reflected out of the cable's core. DSI3D is well suited for studying this type of problem. The cleave intersection with the cylindrical fiber is easily handled using the unstructured and nonorthogonal grid features of DSI3D. Figures 6 and 7 show the nature of the reflected pulses for a centered cleave and an offset cleave, respectively. The differences between the two reflected signals are obvious.

Another new interesting application has been the use of DSI3D to study the behavior of whispering-gallery-mode microdisk lasers. ${ }^{3}$ These novel devices have potential for the integrability and low-power operation required for large-scale photonic circuits. The disks are formed using selective etching techniques in a InP/InGaAsP system to achieve 3- to $10-\mu \mathrm{m}$-dia disks as thin as $500 \AA$ suspended in air or $\mathrm{SiO}_{2}$ on an InP pedestal. Optical confinement within the thin disk plane results in a microresonator with potential for single-mode, ultra-low threshold lasers. Figure 8 shows the 


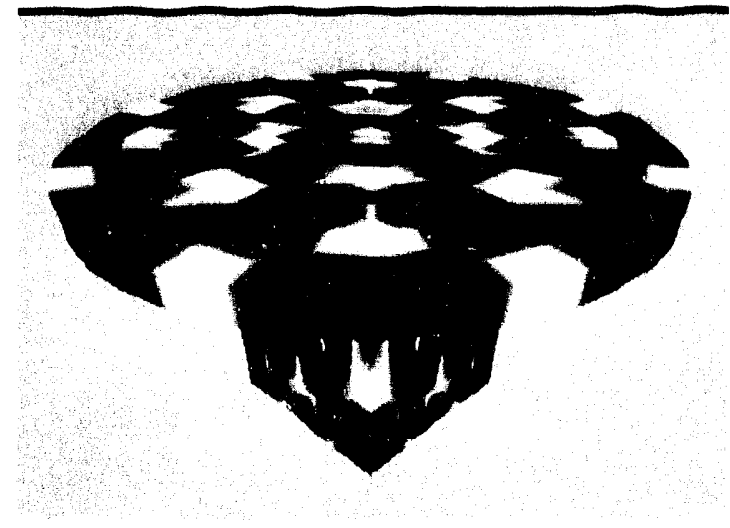

Figure 9. Fieid plot showing the $M=8$ mode for the microdlsk laser and pedestal.

DSI3D grid for the disk and pedestal. The grid for the surrounding medium is not shown. Figure 9 shows the excited $M=8$ mode for the disk and pedestal. Figure 10 shows the structure of the radiated fields in the plane of the disk.

\section{Future Work}

Our DSI3D algorithm and code, together with the ever-more-capable MPP computers, give us a unique opportunity for significant new contributions to 3-D EM modeling. We now have the flexibility and capability to solve problems of a size and detail that were previously unimaginable. We intend to address to a much greater extent some of the areas of application mentioned above. In addition, we plan to add a charged-particle capability to DSI3D, so that these new capabilities will be available to the plasma physics community.

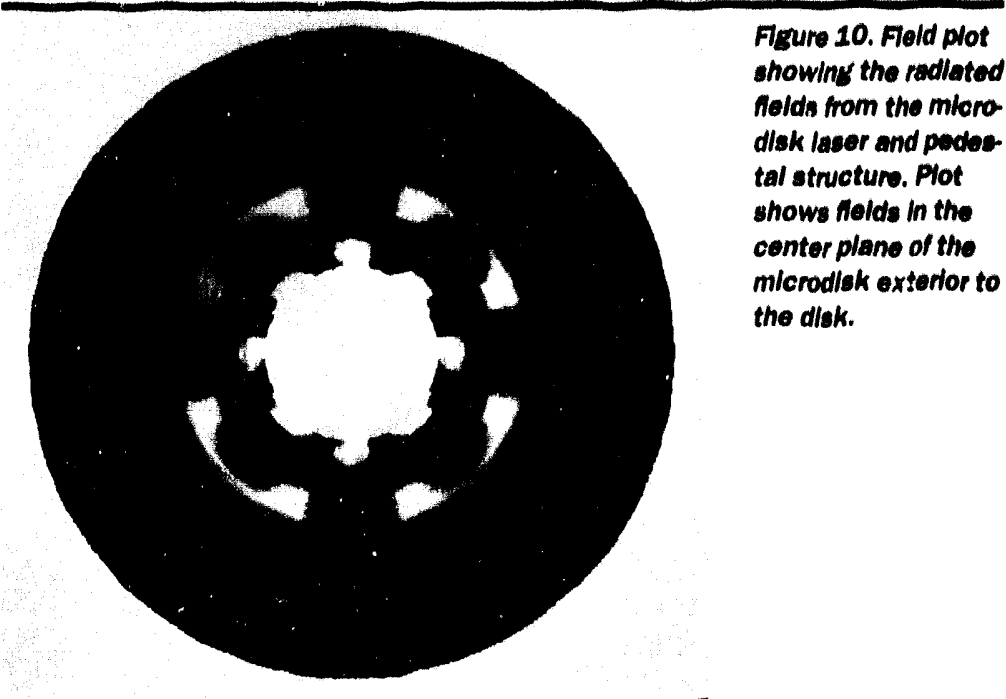

We have attracted the interest of several industrial partners, and Cooperative Research and Development Agreement efforts are underway with these partners to develop specialized versions of DSI3D for use in RCS analysis and for gyrotron design.

1. N.K. Madsen, Divergence Preserving Discrete Surface Integral Methods for Maxzerll's Curl Equations Using Non-Orthegronal Lnstructured Grids, Lawrence Livermore National Laboratory, Livermore, California, UCRL-JC-109787 (1992).

2. H.D. Simon, Computing Systems in Enginetring 2 $(2 / 3), 135(1991)$.

3. S. McCall, A. Levi, R. Slusher, S. Pearton, and R. Logan, Appl. Plyys. Lett. 60 (3), 289 (1992). 


\section{Computational Integrated Photonics}

\author{
Raymond J. Hawkins and \\ Jeffrey S. Kallman \\ Engineering Research Division \\ Electronics Engineering
}

\author{
Richard W. Ziolkowski \\ Department of Electrical and \\ Computer Engineering \\ University of Arizona \\ Tucson, Arizona
}

We have continued our innovative work in computational integrated optics, a field important both to programs at Lawrence Livermore National Laboratory (LLNL) and to industry. Integrated optical device design has been our primary research topic. The results of this project have been applied to device design at LLNL, at Bellcore in Red Bank, New Jersey, and at Hughes. A second leading project, device design code integration and graphical user interface development, has also proved to be of great significance, with our simulation results proving to be of interest to a number of companies.

\section{Introduction}

Computational integrated photonics (CIP) is the area of computational physics that studies the propagation of light in optical fibers and in integrated optical circuits (the photonics equivalent of electronic circuits). The purpose of integrated photonics simulation is to develop the computational tools that will support the design of photonic and optoelectronic integrated devices. These devices will form the basis of all future high-speed and high-bandwidth information-processing systems and are key to the future industrial competitiveness of the U.S. CIP has, in general, two thrusts: (1) to develop predictive models of photonic device behavior that can be used reliably to enhance significantly the speed with which designs are optimized for applications, and (2) to further our ability to describe the linear and nonlinear processes that occur and can be exploited in real photonic devices.

Our efforts in FY-92 have been focused in three general areas: (1) pseudospectral optical propagation codes; (2) linear finite-difference time-domain (FDTD) codes; and (3) nonlinear FDTD codes. This year we have focused on both the development of codes of interest to the integrated optics community, and on packaging these codes in a user-friendly manner, so that they can be used by other researchers in both academic and industrial laboratories. We have developed two new design codes, BEEMER and TSARLITE, with graphical user in- terface (GUI), and have made significant advances in nonlinear FDTD.

As FTDT becomes increasingly popular for the study of integrated optical systems, the need to include material dispersion and nonlinear effects has forced us to examine these issues. We found a particularly convenient way of including linear material dispersion in FDTD calculations, and have funded studies in the inclusion of material nonlinearities in FDTD calculations.

\section{Progress}

Our work in integrated optical device design continues to give us our leading role in the design of integrated optical components both for Lawrence Livermore National Laboratory programs and for U.S. industry. This research is of particular interest, since we have predictive codes that significantly reduce the time required to bring a device from concept to prototype.

Our work with the pseudospectral optical propagation code, called the beam propagation method (BPM), has addressed the issue of understanding optical field evolution in multilayer, integrated guided-wave detector structures. This work, which previously led to the development of extremely short integrated waveguide/photodiodes with high quantum efficiency, has now resulted in the development of the polarization diversity detectior shown in Fig. $1^{1}$ and the coherent receiver she $n$ in Fig. 2.2 


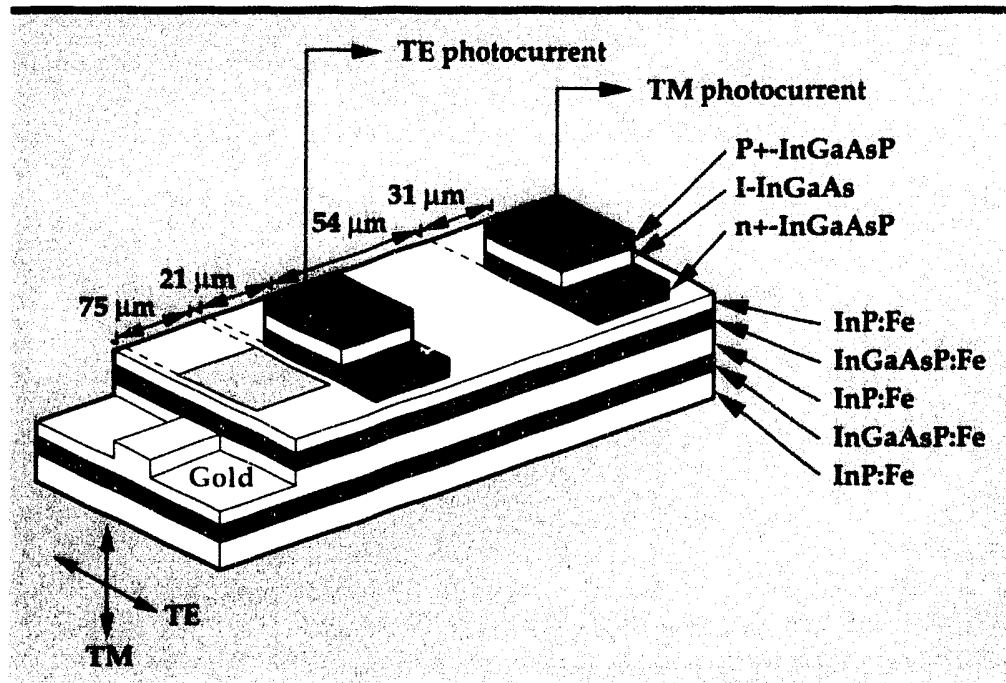

Figure 1. The Bellcore polarization-diversity photodetector that produces two photocurrent outputs proportional to guide-input intensities in each of two orthogonal polarization states. Our device is significantly (a factor of 5 to 20) smaller than previous monolithic realizations of this circuit.

We were also able to help researchers at Hughes understand the operation of their photodetectors, since they were based on a very similar design. Our work with Bellcore was selected as an example of leading work in optical interconnections. ${ }^{3}$

For several years, BPM has been the method of choice for computational physicists studying integrated optical waveguide/device behavior. Unfortunately, the special methods underlying the BPM that made it so efficient also made it difficult for many to code from scratch. Distribution of source code was found, empirically, to be an unsatisfactory alternative, since most codes are writ- ten by and for computational physicists. Consequently, the BPM was often admired from afar by those who would best benefit from a hands-on capability.

To fill the void, we wrote BEEMER, a BPM code with a GUI that allows construction of a device layout, simulation, and optimization, all within the same window structure. The designer can specify a variety of material parameters including gain, loss, and Kerr nonlinearity. Thus, this tool can easily handle design problems from linear photodetectors to all-optical soliton-based switches. BEEMER is written in $C$ and has been compiled successfully on a number of workstations, including SUN, IBM, DEC, and SGI. An illustration of the type of problem that BEEMER can handle is shown in Fig. 3. The manual for BEEMER guides the user through a number of examples drawn from various areas in optics, to acquaint the user with the program. BEEMER and the manual have been released for distribution outside of $L L N L$, and we have installed BEEMER at both academic and industrial sites.

To meet the needs of a variety of photonics device designs, we have continued our development of FDTD as a tool for integrated optical device simulation, extending our previous expertise in pseudospectral-code-based device simulation. Our FDTD work has provided information on a variety of devices that could not be modeled by any existing codes. For example, we have demonstrated the ability to model diffraction gratings and facet reflections. The FDTD treatment of electromagnetic pulse propagation holds much prom-

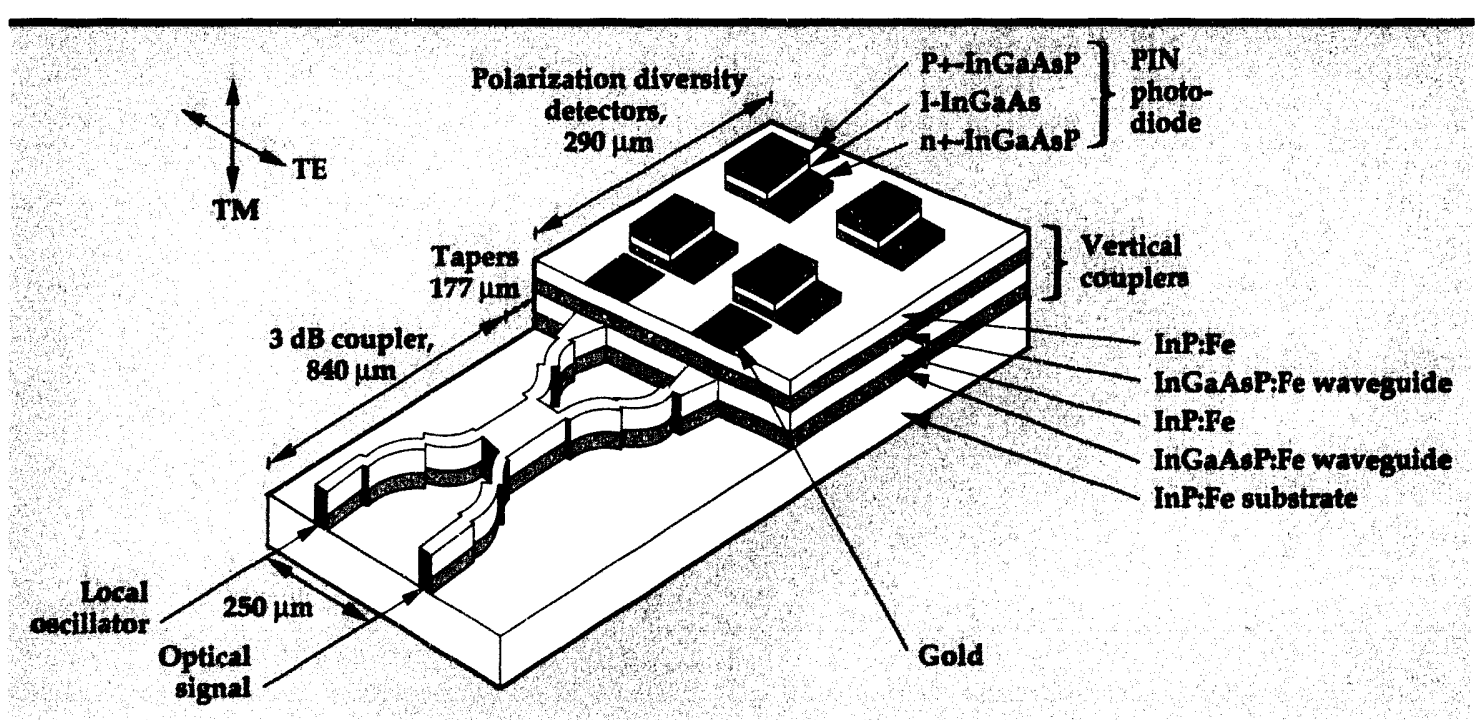

Figure 2. The Bellcore ultracompact, balanced, polarization diversity photodetector. The two detectors corresponding to each polarization state are interconnected for on-chip photocurrent subtraction, which is essential for broadband, balanced operation without microwave hybrids. 
ise for the complete numerical description of integrated optical device behavior, where reflections and/or coherent effects are important. The recent application of FDTD to problems in integrated optics $4,5,6$ has indicated that electronic dispersion must be included to treat realistically the broadband behavior of integrated optical devices. The inclusion of material dispersion (electronic or magnetic) in FDTD calculations has historically been quite limited. The first formulation of broadband dispersion in FDTD was presented in a pioneering paper ${ }^{7}$ that demonstrated that if the electronic susceptibility was expanded as a series of exponentials, the treatment of dispersion could be reduced to a recursive update. The incorporation of this update, however, requires a substantial rewriting of the standard electric-field update equations.

More recently, others 8,9 demonstrated a different formulation of the linear problem, explicitly solving the equation of motion for the polarizability using finite-differencing. This alternative formulation has been extended ${ }^{10}$ to nonlinear optical propagation. In our work, we have exploited a simple causality argument that enabled us to write dispersion as a simple, recursive, additive term in the common electric-field update equations. This is of particular interest, since it enabled the treatment of dispersion in a large number of existing FDTD design codes with minimal computational modification.

While there has been a great increase in integrated optical devices for which only a solution of Maxwell's curl equations will suffice, ease of use has not been the hallmark of these codes. To meet this need and to provide ease of user access, we have written TSARLITE9: a two-dimensional FDTD code with a fully integrated GUI. [TSAR is

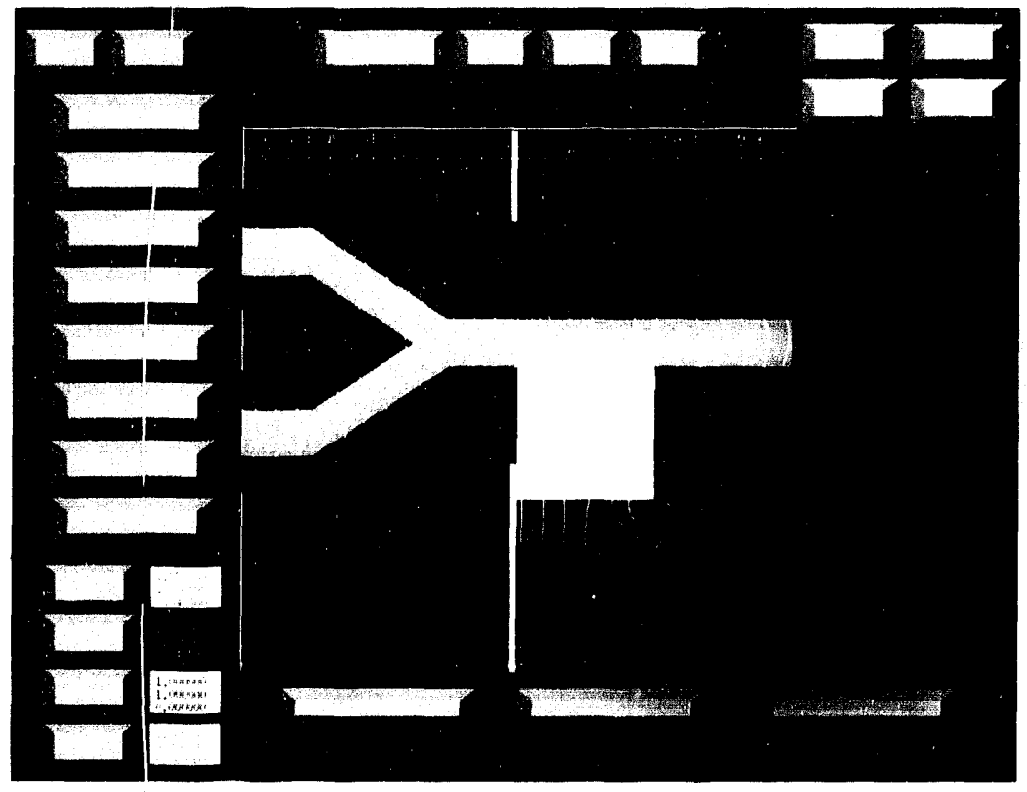

Figure 3. An all-optical switch based on spatial solitons. Light coming in from the left is combined into a waveguide that is placed next to a nonlinear medium. If the combined intensity is great enough (as shown), then the evanescent field in the nonlinear medium is strong enough to form a spatial soliton that splits off and is subsequently captured by the lower arm.

an established, three-dimensional (3-D), FDTDcode with limited GUI application.] TSARLITE has been constructed with the integrated optics community in mind, and thus has desirable features such as the ability to launch spatially and temporally shaped pulses, and our latest dispersion model. An example of the type of problem that TSARLITE can handle is shown in Fig. 4. Unlike BEEMER, TSARLITE does not yet have a manual and has not yet been released for use outside of LLNL. We anticipate that this will happen in the coming year.

With the continuing and heightened interest in nonlinear semiconductor and optically integrated
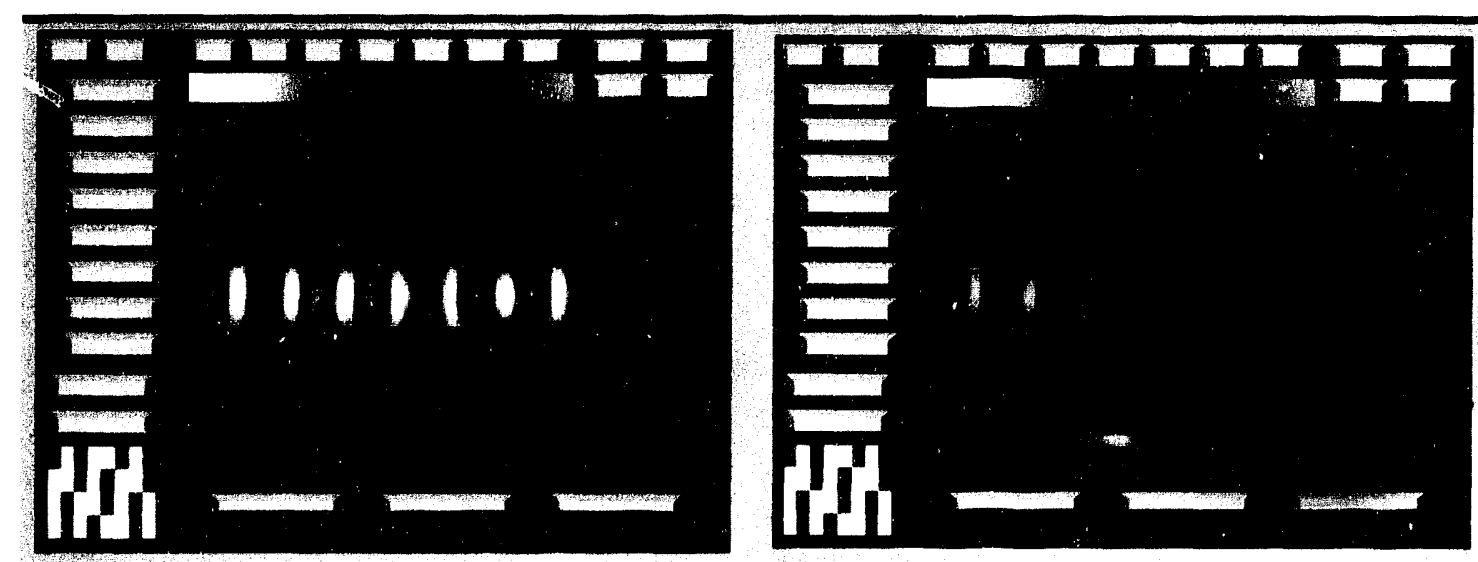

Figure 4. An sptical crossbar element. On the left, the element is in transmit mode, but the degree of confinement of the light in the waveguide leads to significant loss in the cross region. With the mirror in place (right), the light is coupled into the waveguide, but the offset of the mirror from an ideal position results in some scattering losses. 
devices, more accurate and realistic numerical simulations of these devices and systems are in demand. To date, most of the modeling of pulse propagation in and scattering from nonlinear media has been accomplished with one-dimensional, scalar models. These models have become quite sophisticated; they have predicted and explained many of the nonlinear as well as linear effects in present devices and systems. Unfortunately, they cannot be used to explain many observed phenomena, and are probably not adequately modeling linear and nonlinear phenomena that could lead to new effects and devices. Vector and higher dimensional properties of Maxwell's equations that are not currently included either in existing scalar models or in more detailed material models, may significantly impact the scientific and engineering results. Moreover, because they are limited to simpler geometries, current modeling capabilities are not adequate for linear/nonlinear optical-component engineering design studies. The successful development of general, linear, and nonlinear electromagnetic modeling capabilities will significantly impact the concept and design stages associated with novel linear and nonlinear phenomena and the resulting optical components.

We have developed the first multi-dimensional, full-wave, vector solutions to Maxwell's equations for problems describing the interaction of ultra-short, pulsed beams with a nonlinear Kerr material having a finite response time.12 These solutions have been obtained with a nonlinear finite-difference time-domain (NL-FDTD) method developed by investigators at the University of Arizona. This NL-FDTD method combines a nonlinear generalization of a standard, FDTD, fullwave, vector, linear Maxwell's equation solver, with a currently used phenomenological time relaxation (Debye) model of a nonlinear Kerr material. In contrast to a number of recently reported numerical solutions of the full-wave, vector, timeindependent Maxwell's equations and of vector paraxial equations, the FDTD approach is a timedependent analysis that accounts for the complete time evolution of the system, with no envelope approximations. Nonlinear, self-focusing numerical solutions in two space dimensions and time that are obtained with this NL-FDTD method, as well as related NL-FDTD results for normal and oblique incidence, nonlinear interface problems, have been investigated. Although these basic geometries are straightforward, the NL-FDTD approach can readily handle very complex, realistic structures.

The chosen sample TE and TM nonlinear optics problems highlight the differences between the scalar and the vector approaches, and the effects of the finite response time of the medium. The NL-FDTD method is beginning to resolve several very basic physics and engineering issues concerning the behavior of the full electromagnetic field during its interaction with a self-focusing medium. In particular, using the NL-FDTD approach we have (1) shown the first back reflections from the nonlinear self-focus; (2) discovered optical vortices formed in the trailing wakefield behind the nonlinear self-focus; (3) identified that the longitudinal field component plays a significant role in limiting the self-focusing process; (4) performed the first complete full-wave, vector treatment of both the TM and TE models of an optical diode (linear/nonlinear interface switch); (5) characterized the performance of an optical diode to single-cycle pulsed Gaussian beams, including the appearance of a nonlinear GoosHänchen effect, the stimulation of stable surface modes, and the effects of a finite response time of the Kerr material; (6) shown definitively that the linear/nonlinear interface does not act like an optical diode for a tightly focused, single-cycle pulsed Gaussian beam; and (7) characterized the performance of some basic linear/nonlinear slab waveguides as optical threshold devices.

In all of these analyses, we have identified the role of the longitudinal field component and the resulting transverse power flows in the associated scattering/coupling processes.

\section{Future Work}

We will continue our efforts in the design of novel integrated optical devices, both for LLNL programs and for industry. It is our intention to transfer BEEMER and TSARLITE to industry. Our development of linear FDTD applications to integrated optics will be extended to 3-D structures, and our studies of NL-FDTD will continue in the area of nonlinear waveguides and couplers.

1. R.J. Deri, R.J. Hawkins, E.C.M. Pennings, C. Caneau, and N.C. Andreadakis, Appl. Phys. Lett. 59 (15), 1823 (1991).

2. R.J. Deri, E.C.M. Pennings, A. Scherer, A.S. Gozdz, C. Caneau, N.C. Andreadakis, V.Shah, L. Curtis, R.J. Hawkins, J.B.D. Soole, and J.-I. Song, Photonics Tech. Lett. 22, 1238 (1.992).

3. R.J. Deri, E.C.M. Pennings, and R.J. Hawkins, $O p$ tics and Photonics Nezus (December, 1991).

4. S.T. Chu and S.K. Chaudhuri, J. Lightwove Teclinol. LT-7, 2033 (1989). 
5. S.T. Chu, Modelling of Guided-Wave Optical Structures by the FDTD Method, Ph.D. Thesis, University of Waterloo (1990).

6. S.T. Chu and S. Chaudhuri, IEEE Trans. Micrownu' Theory Tech. 38, 1755 (1990).

7. R. Luebbers, F.P. Hunsberger, K.S. Kunz, R.B. Standler, and M. Schneider, IEEE Trans. Electronnagn. Compat. EMC-32, 222 (1990).

8. C.F. Lee, R.T Shin, and J.A. Kong, PIER4 Progress in Electromagnetics Research, J.A. Kong (Ed.), Elsevier Science Publishing Company, Inc. (New York), 415, 1991.
9. R.M. Joseph, S.C. Hagness, and A. Taflove, Opt. Litt. 16, 1412 (1991).

10. P.M.Goorjianand A. Taflove, Opt. Lett. 17, 1412(1992).

11. R.W.Ziolkowski andJ.B.Judkins, "Propagation Characteristics of Ultra-Wide Bandwidth Pulsed Gaussian Beams," accepted for publication in /OSA A (November 1992).

12. R.W.Ziolkowski and J.B. Judkins, "Full-Wave Vector Maxwell Equation Modeling of the Self-Focusing of Ultrashort Optical Pulses in a Nonlinear Kerr Medium Exhibiting a Finite Response Time," IOSA B 10, 186(1993). 


\section{Analysis of High-AveragePower, Millimeter-Wave Microwave Components and Induction Linear Accelerator Modules}

\author{
Clifford C. Shang and \\ John F. DeFord \\ Engineering Research Division \\ Electronics Engineering
}

\author{
Malcolm Caplan \\ Magnetic Fusion Energy Program
}

In FY-92, we analyzed high-average-power, millimeter-wave microwave components in rf systems for heating fusion plasmas and induction linear acceleratur mociules for heavy ion fusion. The electrical properties of these structures were studied using time-dependent electromagnetic field codes and detailed material models.

We modeled gyrotron windows and gyrotron amplifier sever structures for transverse electric modes in the 100- to $150-\mathrm{GHz}$ range, and computed the reflection and transmission characteristics from the field data. Good agreement between frequency domain codes and analytic results has been obtained for some simple geometries. We describe results for realistic structures with lossy dielectrics and the implementation of microwave diagnostics.

For the modeling of induction accelerators (electron machines), understanding the coupling of the beam to the cavity is of fundamental importance in estimating the effects of transverse beam instabilities. Our accelerator modeling work focused on examining the beam-cavity interaction impedances (impulse response of cavity) for subrelativistic beams in drivers for heavy ion fusion, to better underst r nd longitudinal ( $n=0$, monopole) and transverse $(n=1$, quadrupole) beam instabilities. Results for simple segmented cell configurations show that the pulse power system and induction cores are largely decoupled from wakefields.

\section{Introduction}

Robust algorithms for the solution of Maxwell's equations in the time domain have been known for some time. ${ }^{1,2}$ Since 1966, specializations of these algorithms to include more sophisticated boundary conditions ${ }^{3,4}$ and detailed material models ${ }^{5,6}$ have allowed the application of the basic numerical techniques to interesting problems. Further, recent algorithm developments 7,8 for Maxwell solvers on conforming meshes now allow high geometrical fidelity that may be required for a certain class of problems.

\section{Progress}

In FY-92, we examined two sets of problems. The first set involves high-average-power milli- meter-wave ( $\mathrm{mmw}$ ) structures; the second involves induction linear accelerator cells. The principle features in modeling the mmw structures are the launching of modes, the modeling of lossy dielectrics, and the development of microwave diagnostics. The fundamental aspects of modeling the heavy-ion induction cells include implementing realistic, magnetically dispersive material models and computing subrelativistic wake potentials. ${ }^{9}$

\section{Modeling mmw Components}

The use of high-power microwaves to heat the plasma in a magnetic fusion energy (MFE) reactor at the electron-cyclotron resonance can yield a number of benefits, such as bulk-heating and preionization of the plasma; reaction startup; and instability suppression. The use of electron-cyclo- 
tron heating $(\mathrm{ECH})$ in tokamak and stellerator reactors has been studied in many significant MFE experiments, including C-mod at the Massachusetts Institute of Technology and DIII-D at General Atomics in the U.S.; Compass at Culham, England; T-10 at the Kurchatov Institute, Russia; and the Heliotron at Nagoya, Japan.

Operating parameters of interest for ECH applications include frequencies in the 140- to $250-\mathrm{GHz}$ range and output power in the vicinity of $1 \mathrm{MW}$ per bottle. ${ }^{10}$ Currently, the fixed-frequency mmw source available for use in the 1-MW range is the gyrotron. Understanding the microwave properties of high-average-power rf structures is crucial to the design of gyrotron tubes and amplifier devices. Dissipation of the rf (ohmic loss) and excessive mode conversion are often limiting factors in the performance and robustness of the overall device. These issues and others pertaining to $\mathrm{mmw}$ devices can be investigated using timedomain electromagnetic (EM) field codes. ${ }^{11}$ An advantage of simulation in the time domain is that EM characteristics can be obtained over a wide bandwidth from a single calculation. Excitation of the frequencies of interest can be obtained by launching modulated pulses driven by magnetic currents. A general field code such as AMOS ${ }^{12}$ can be used to launch the prescribed modes at the frequency or frequencies of interest to examine mmw component performance by numerical integration of Maxwell's equations.

Mode Launching. Gyrotron oscillators operate with whispering gallery (WG) modes, for which the radial mode number greatly exceeds the axial mode number. Thus, most of the rf is distributed near the beam-pipe wall. As the mode propagates near the window, the modes couple into gaps in the window assembly, leading to mode conver-

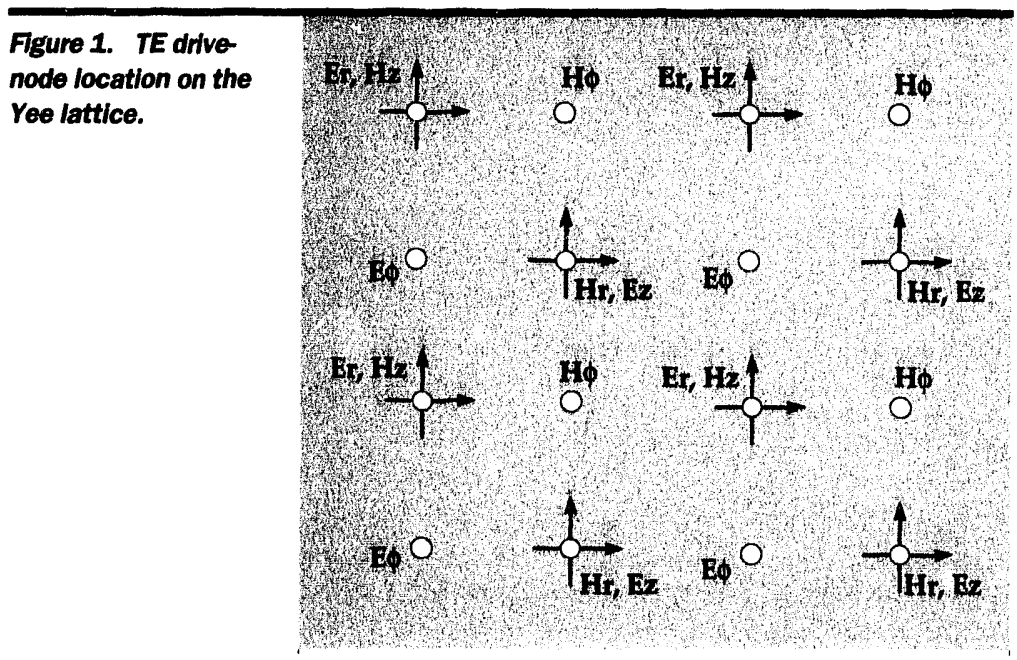

sion. To model gyrotron components requires the launching of transverse electric modes $\left(T E_{n, m}\right)$. This is accomplished by driving magnetic currents over the beam-pipe aperture.

To describe the location of the TE drive-nodes, we rewrite the EM time-dependent curl equations:

$$
\begin{gathered}
\nabla \times \mathbf{H}=\sigma \mathrm{E}+\varepsilon \frac{\partial \mathrm{E}}{\partial t}+\mathrm{J}_{s} \\
\nabla \times \mathrm{E}=-\mu \frac{\partial \mathbf{H}}{\partial t}-\mathbf{K}_{s}
\end{gathered}
$$

in the integral form

$$
\begin{gathered}
\oint \mathrm{H} \bullet d \ell=\iint\left(\sigma \mathrm{E}+\varepsilon \frac{\partial \mathrm{E}}{\partial t}+\mathrm{J}_{s}\right) \cdot d \bar{A} \\
\oint \mathrm{E} \bullet d \ell=-\iint\left(\mu \frac{\partial \mathrm{H}}{\partial t}+\mathrm{K}_{s}\right) \cdot d \bar{A} .
\end{gathered}
$$

$\mathrm{K}$ source components are co-located with $\mathrm{H}$ field components on the Yee lattice. ${ }^{1}$ Referring to Fig. 1 and Eq. 4, one can see that driving the $K_{r}$ component of the magnetic current will excite the proper $\mathrm{H}_{\mathrm{r}}, \mathrm{H}_{\mathrm{z}}$ and $\mathrm{E}_{\phi}$ fields. Similarly, $\mathrm{K}_{\phi}$ currents excite $\mathrm{E}_{\mathrm{r}}$ and $\mathrm{H}_{\phi}$ field components.

The prover spatial variation of magnetic currents required to obtain propagating $W G \mathrm{TE}_{22,2}$ modes are the Bessel function $J_{22}(x)$ out to the second zero, and its derivative $\mathrm{J}_{22}(\mathrm{x})$, which directly drive $K_{\phi}$ and $K_{r}$, respectively. The amplitude distribution in time can be a modulated pulse to obtain the required frequency content (Fig. 2).

Field diagnostics for computing the voltage standing wave ratio (VSWR) were incorporated into AMOS by sampling electric fields at 'numeri$\mathrm{cal}^{\prime}$ probes and computing the VSWR directly from the field values. If $\boldsymbol{F}[\mathrm{f}(\mathrm{t})]$ denotes the forward Fourier transform, then the VSWR can be computed from the field data by first computing the reflection coefficient (no mode conversion),

$$
\Gamma=\sqrt{1.0-\left(\mid \frac{\Im\left[e_{s m p}(t)\right]}{\mathfrak{I}\left[p_{\bmod }(t)\right]}\right)^{2}},
$$

where $e_{\text {smp }}(t)$ is the sampled electric field on the 'downstream' side of the window, and $p_{\text {mod }}(t)$ is the modulated pulse in time. The VSWR is computed according to the definition VSWR $=(1.0$ $+\Gamma) /(1.0-\Gamma)$.

Results of mmw: High-Power rf Window Analysis and Gyrotron Amplifier Sever. Presently, gyrotrons operate in the $100-$ to $140-\mathrm{GHz}$ and $\sim 1$-MW regime. Future performance requirements 


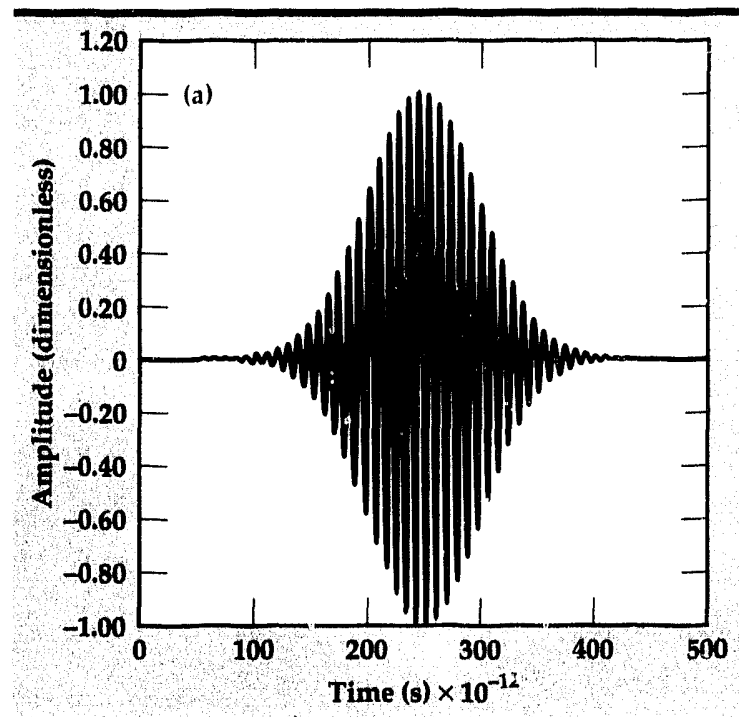

will increase power levels to the multi-megawatt range with frequencies approaching $250 \mathrm{GHz}$. In this scenario, $\mathrm{mmw}$ components will be placed under severe mechanical and thermal stress. Until now, less demanding performance constraints have rendered non-ideal component effects less important. However, understanding these effects is now critical to the operation of the device.

We now examine high-order mode scattering caused by various rf window geometries at the exit of the gyrotron. The VSWR associated with the window can be determined over a broad spectrum of frequencies, using data from a single timedomain run with the technique described in the previous section.

In Fig. 3, we find good agreement between

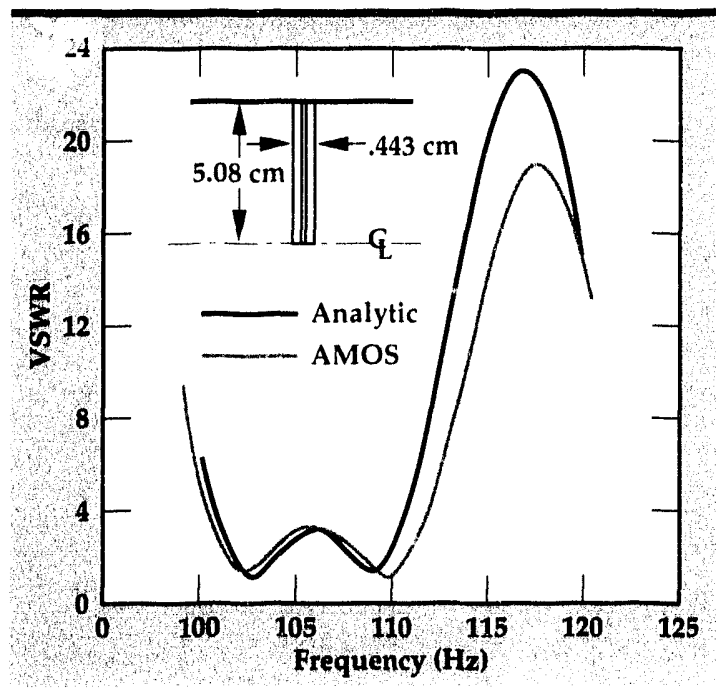

Fgure 3. VSWR for idealized 110-GHz bandpass, from ana Iytic calculations and from AMOS. The inset shows the gyrotron window geometry.

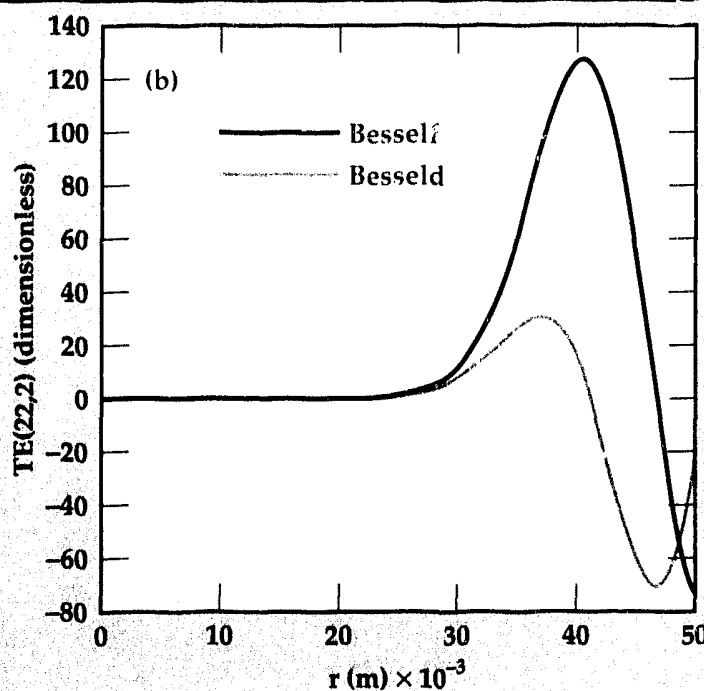

Figure 2. Launching $T E_{22,2} W G$ modespatial and temporal magnetic drive function.
AMOS and analytic values ${ }^{13}$ for the VSWR of a three-layer $\mathrm{rf}$ window. The gyrotron window geometry includes a beam-pipe radius of $5.08 \mathrm{~cm}$ with the longitudinal extent of the window at $0.443 \mathrm{~cm}$. The window material has $\varepsilon_{\mathrm{r}}=9.387$, and the dielectric cooling fluid has $\varepsilon_{\mathrm{r}}=1.797$. A small difference between the AMOS and frequency code results is evident, caused by a minor variation in window element thicknesses resulting from the use of a regular grid in AMOS.

The gyrotron window structure is grown from a sapphire crystal. The window assembly is expensive and difficult to fabricate, but more realistic window geometries cannot be easily treated analytically. In Fig. 4, a realistic window structure with the 'coolant reservoir' is modeled. Compared

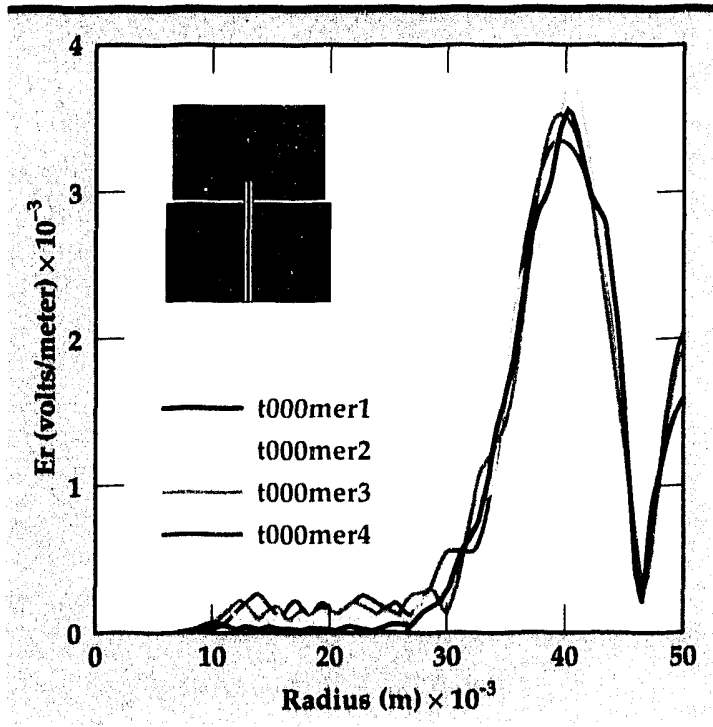

Flgure 4. Radlal field profle at varying longitudinal loca tions for reallstic gyrotron if window structures. The inset shows the window geometry. 
Fgure 5. Sever performance for berrylia $60 / 40$.

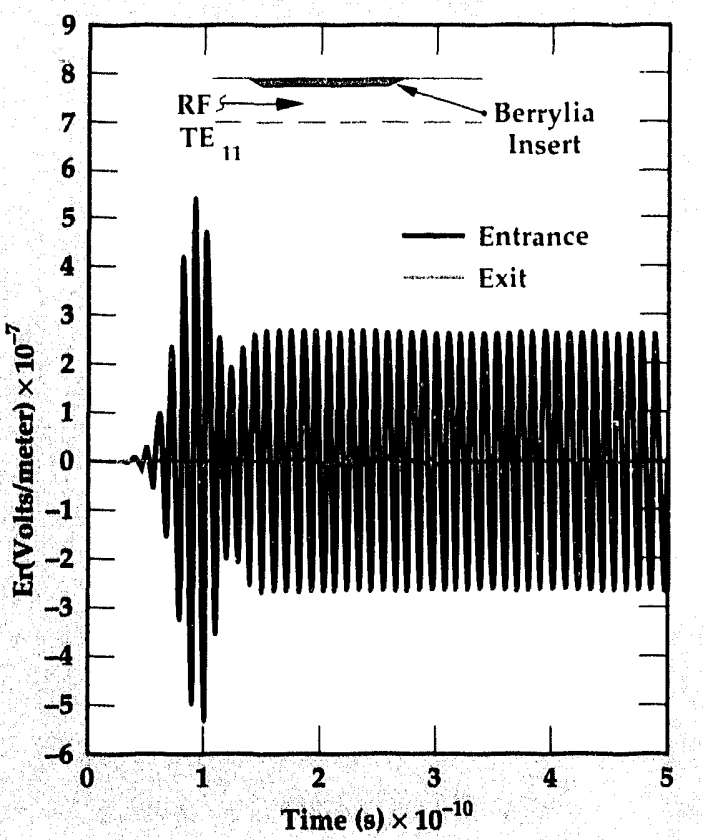

to the idealized window, the electric fields near axis highlight coupling to modes through the rf window near the beam-pipe wall. At the multimegawatt range, this amount of rf may be significant. However, the exact level of power per mode awaits further analysis.

We performed a second set of calculations in which we examined wave propagation through a microwave sever, a device for stopping or absorbing microwave energy while allowing a charged

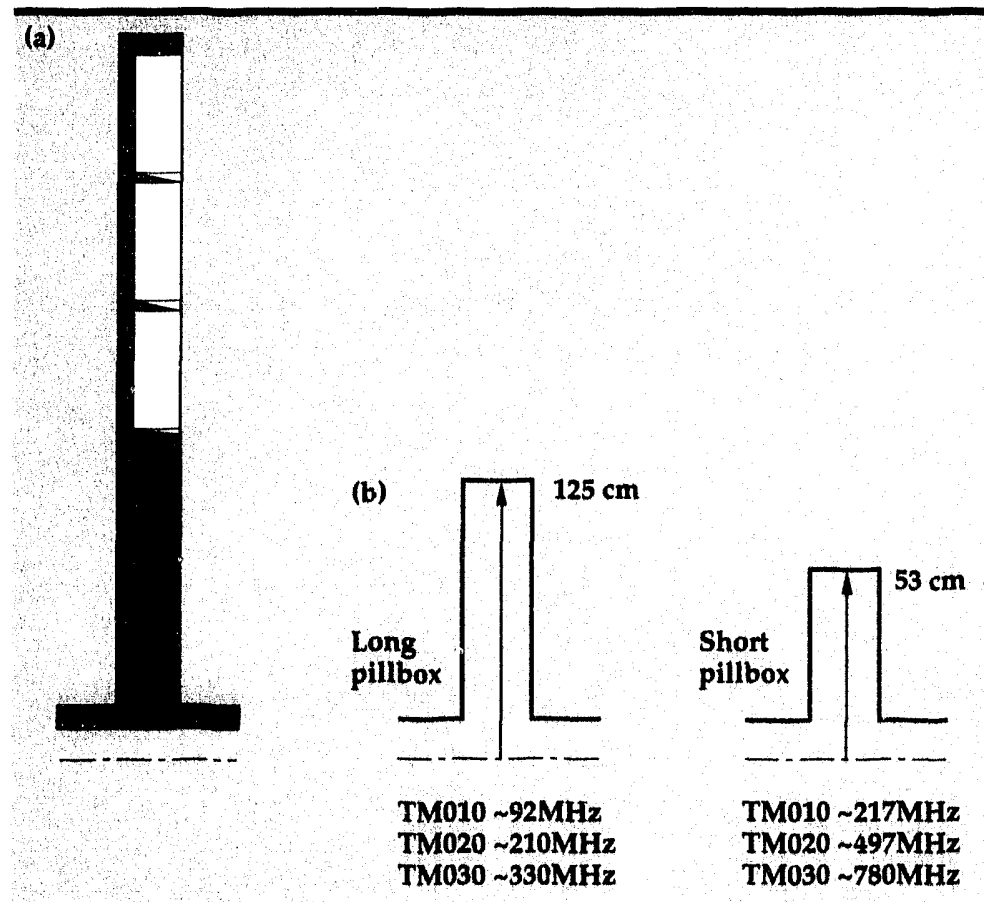

Figure 6. Segmented induction core geometry Illustrating 'Iong' and 'short' pillbox regions. particle beam to pass undisturbed. Of interest is the understanding of the absorption in the lossy dielectric insert (see Fig. 5) for a variety of lossy if mixtures. The beam-pipe radius is $0.95 \mathrm{~mm}$, which is near the cutoff radius. As before, TE 11 modes were launched by driving magnetic currents at the sever aperture. The material conductivity characteristics for five berrylia mixtures were obtained from the available experimental data at $12 \mathrm{GHz}$. Two materials, berrylia $80 / 20$ and berrylia $60 / 40$, are representative: the dielectric constants $\mathrm{K}^{\prime}$ for berrylia $60 / 40$ and $80 / 20$ are 49.54 and 17.81 , respectively, and the loss tangents are 0.72 and 0.22 , respectively. ${ }^{14}$

AMOS predicted $\sim 40 \mathrm{~dB}$ attenuation for a 95- $\mathrm{GHz} T \mathrm{TE}_{11}$ mode propagating toward the sever for the berrylia 60/40 mixture. In comparison, unacceptably low rf absorption characteristics for the other berrylia mixtures (Fig. 5) were evident. In the limit, when the conductivity is large (berrylia 60/40), the relevant diameter is not that of the beam-pipe, but instead it is the diameter inside the sever section. With cutoff given by $\lambda_{c}=2 \pi \mathrm{a} / 1.84$, a $\mathrm{TE}_{11}$ mode at $95 \mathrm{GHz}$ is well below cutoff, and the fields will be attenuated. This set of calculations can be repeated when updated berrylia measurements in the $\sim 100-\mathrm{GHz}$ range are available. For the previous class of modeling problem, we plan to examine simulation issues such as the launching of waveguide modes near cutoff. Further, the taper of the lossy section was initially limited to a minimum of $5^{\circ}$ because of numerical limitations of a shallow-angle staircasing of the mesh. The conforming mesh algorithm in CG-AMOS ${ }^{15}$ will allow exact boundary representation, and thus any shallow taper.

\section{Modeling Induction Linear Accelerator Modules}

We have modeled the beam-cavity interaction impedances for induction linear accelerator cells for heavy ion fusion. The induction cell works conceptually much like a 1:1 transformer with the beam as secondary. The primary one-turn loop has a pulsed voltage $V$ applied to it. The secondary loop around the core will have a voltage induced across its terminals that is the same as the primary voltage, i.e., from Faraday's law, V $=\mathrm{AdB} / \mathrm{dt}$. The core consists of wound metallic glass (Metglas), which has good dB/dt characteristics (1 to $5 \mathrm{~T} / \mu \mathrm{s})$. In the three-segment core configuration proposed by Lawrence Berkeley Laboratory (LBL), each core is fed in parallel. The secondary loop encloses all three cores, providing 


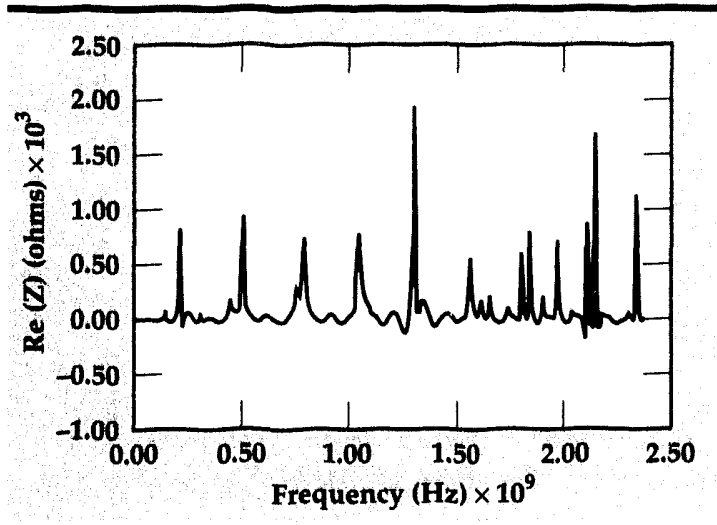

Figure 7. Impedance spectrum for the base induction module.

$3 \mathrm{AdB} / \mathrm{dt}$ at the accelerating gap. The equivalent single core configuration would require either three 1-V accelerating gaps or a 3-V pulse power system. Another advantage of the segmented configuration is that one part of the core will not saturate before any other part.

In FY-92, we concentrated on undeistanding the segmented cell geometry and multicell accelerating modules for subrelativistic heavy ions from a beam-cavity coupling point of view .

Results from Accelerator Modeling. For the base case (Fig. 6), the gap width is $1.5 \mathrm{crn}$, the radial length is $1.25 \mathrm{~m}$, and the overall cell width is $10 \mathrm{~cm}$. In Fig. 2, the Fourier transform of the impulse response (wake potential) ${ }^{9}$ of the cavity due to charge bunch transiting the accelerating gap shows the beam-cavity coupling (interaction impedances) for the monopole fields.

The dependence of the impedance as a function of $v$, the charge velocity, goes as $\operatorname{sinc}^{2}(\omega d / 2 v)$ (transit time factor), ${ }^{16}$ where $\omega$ is the angular frequency and $d$ is the gap width. We can see, in fact, that the modes at $217 \mathrm{MHz}$ and $497 \mathrm{MHz}$ correspond closely to the $\mathrm{TM}_{010}$ and $\mathrm{TM}_{120}$ modes of the short pillbox geometry, and the weaker coupling of the $92-\mathrm{MHz}$ mode corresponds to the $\mathrm{TM}_{010}$ mode of the 1.25-m radial line (Fig. 7).

For this simplified model, we can see that the dominant feature is the gap width. To determine if the segmented core has good damping features, we opened the gap width to $4.5 \mathrm{~cm}$. The field calculations (Fig. 8) show low $Q$ resonances $(Q$ $=3.91$ ) correspor ding to the 1.25-m (long-pillbox modes) radial line.

The final set of results to be discussed involves the stacked accelerating modules (Fig. 9a). In the absence of inter-cell interactions, it is expected that the impedance of a single cell will add in series.

The result (Fig. 9b) shows that this is indeed the case. Similar simulations were performed for the dipole modes $\left(\mathrm{TM}_{1 \mathrm{~mm}}\right)$ to study the possible impact of beam break-up instabilitylth in heavy ion drivers. These impedance calculations, coupled with analytic and calculational results from the BREAKUP beam dynamics code, indicate controllable beam break-up modes. ${ }^{17}$

\section{Future Work}

Field calculations show that for the current, heavy-ion, linear accelerator cell configuration, the pulse power system and accelerating cores are largely decoupled from wakefields. Although this idealized cell has a high impedance-geometry figure of merit, schemes to lower the $Q$ of the lowerorder modes can be developed. We will continue our work to model the fully three-dimensional, multi-beam-pipe cell as proposed by LBL for the Induction Linac Systems Experiments. We intend to develop detailed anisotropic, dispersive media models of Metglas in the coming year. We also will be involved in research on designs for the nextgeneration induction accelerator for radiography. In the latter work, all cavity modeling results will be incorporated into beam dynamics codes, with the goal being end-to-end simulations leading to dosage estimates.

For high-average-power mmw components, we have shown how application of field codes can be used to analyze complex geometrical aspects that are not amenable to analytical techniques. TE modes may be launched in a beam-pipe by use of the dual $\mathrm{K}$ term (magnetic current) in the Faraday-Maxwell equation. TM modes may be launched using a similar dual technique. Since the rf impinging on the window (110-GHz tube) is in the WG mode, it remains to be seen if the approach for extracting usable modes will involve either (1) converting WG to usable modes external to the

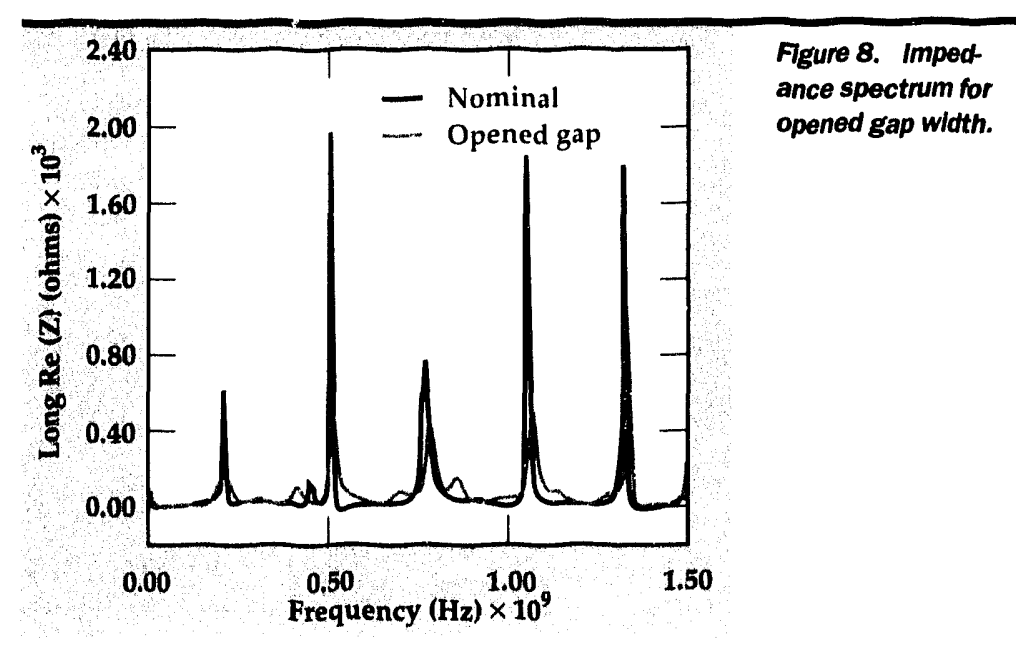




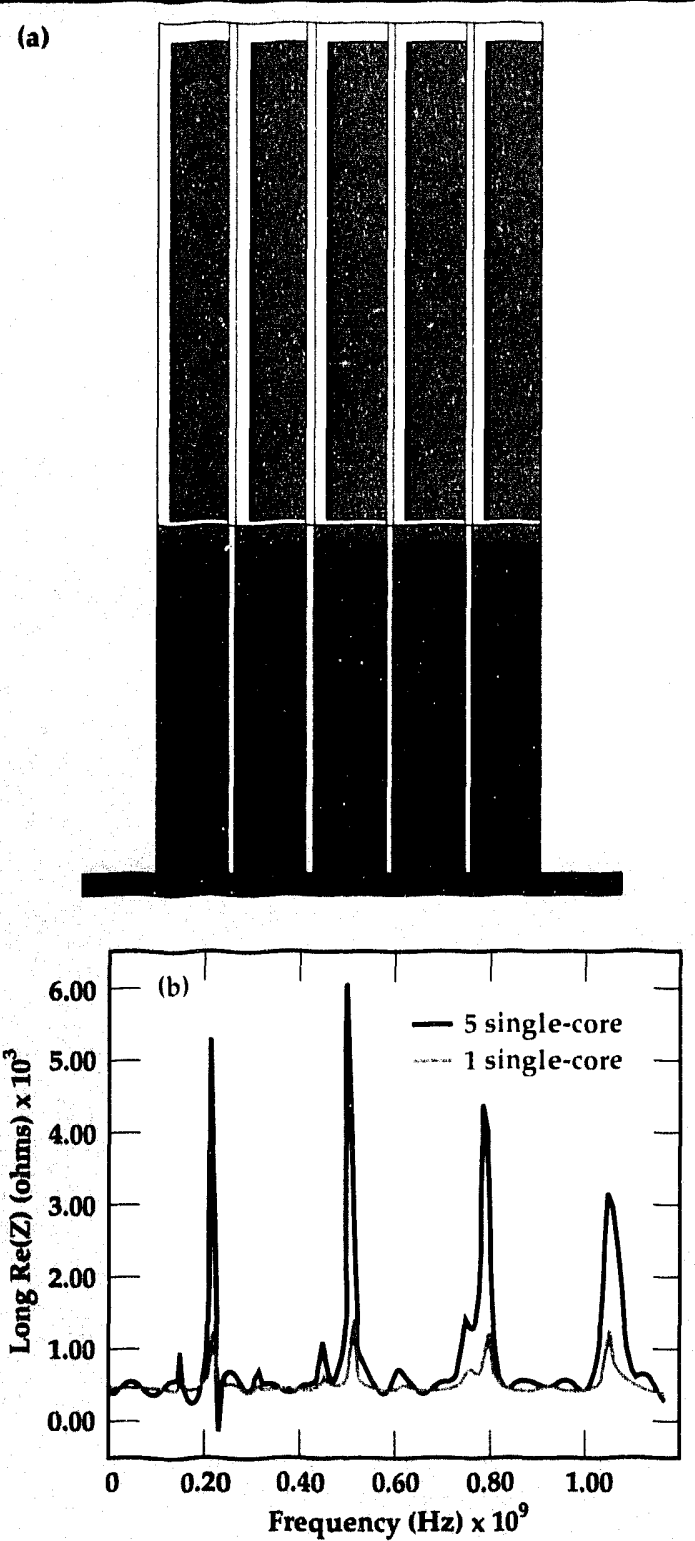

Figure 9. Longitudinal impedance for multi-cell accelerating module.

window or (2) designing a window that can allow a Gaussian power distribution for long pulse operation.

Our future work will also involve the use of unstructured meshes to simulate the important aspects of high-bandwidth ri windows by detailed modeling of window surfaces. We will also implement the diagnostics that will derive mode information directly from the field data.

Our work in $\mathrm{mmw}$ has been formalized in a two-year, \$2.8 M Cooperative Research and Development Agreement with Varian Associates, Inc., Microwave Products Tube Division, of Palo Alto, California and is funded by the Defense Systems and Engineering Directorates at Lawrence Liver- more National Laboratory (LLNL) for FY93 and FY94. We will be examining new mmw circuit concepts, input/output couplers, water loads, and lossy drift tube sections, as well as continuing to model high-average-power windows (fixed frequency and wideband), using field codes.

\section{Acknowledgements}

Stimulating discussions with Drs. J.J. Barnard and G.J. Caporaso (LLNL), and W. DeHope (Varian) are gratefully acknowledged.

1. K.S. Yee, IEEE Trans. Antemmas Propng. AP-14, 302 (1966).

2. A. Taflove and G. Brodwin, IEEE Trmes. Microinn't' Theory and Tedminte's MTT-23, 623 (1975).

3. G. Mur, IEEE Trans. Electromagnetic Compat. EMC-23, $1073(1981)$.

4. K.K. Mei and J. Fang, IEEE Trans. Antemuns Propang. AP-40, 1001 (1992).

5. J.F. DeFord, and G. Kamin, Application of Limear Magnetic Loss Motel of Firrite to lnduction Canity Simulation. Proc of 1990 Linenr Accilerntor Conf. (Aibuquerque, New Mexico), (September 14, 1990).

6. R. Luebbers et al., IEEE Trans. Antennus Propay. AP-32, $222(1990)$.

7. N.K. Madsen, A Dizergence Preserving Discrete Surface lutegration Algorithm for Time-Dommin Moxicell Equations, Lawrence Livermore National Laboratory, Livermore, California, UCRL-JC 109787 (1993).

8. N.K. Madsen and R.W. Ziolokowski, Electrommirynetics $10(1,2), 127$ (1990).

9. J.F. DeFord and G.D. Craig, Part. Accol, 37-38, 111, (1992).

10. W.H. Urbanus, R.W.B. Best, A.G. Verhoeven, and M.J. van der Wiel (FOM-Instituut voor Plasmafysica, Nederland); M. Caplan (Lawrence Livermore National Laboratory, Livermore, California); V. Bratman and G. Denisov (Institute of Applied Physics, Russia); and A.A. Varfolomeev (Kurchatov Institute, Russia), "A 1-MW Free Electron Maser for Fusion Application," Proc. Third European Particle Accelerntor Conf. (Berlin, Federal Republic of Germany), (March 24-28, 1992).

11. M. Caplan, Application of AMOS Code to Design of Complex Structures for Selection Microunare Absorption and Transmission, Lawrence Livermore National Laboratory, Livermore, California, UCRL-ID-109254 (1991).

12. J.F. DeFord, G.D. Craig, and R.R. Mcleod, "The AMiOS Wakefield Code," Proc. Conf. Computer Conte's and the Linear Accelerator Commmunity (Los Alamos, New Mexico), 265 (January 22-25, 1990).

13. J.M. Neilson, P.E. Latham, M. Caplan, and W.G. 
Lawson, IEEE Trans. Microwene Theory and Techniques 37, 1165(1989).

14. W. DeHope, Private communication (April 1992).

15. C.C. Shang and J.F. DeFord, "Modified-Yee Field Solutions in the AMOS Wakefield Code," Proc. 1990 Linear Accelerator Conf. (Albuquerque, New Mexico), (September 14, 1990).
16. R.J. Briggs, D.L. Birx, G.J. Caporaso, V.K. Neil, and T.C. Genoni, Part. Accel. 18, 41 (1985).

17. G.J. Caporaso, "Transverse Instability in a Heavy Ion Fusion Induction Linac," Proc. Longitutinal Instnbility Workshop (Berkeley, California), (February 1992). 


\title{
Electromagnetic Modeling and Experiments for Dispersive Media
}

\author{
Scott D. Nelson and \\ Carlos A. Avalle \\ Defense Sciences Engineering Division \\ Electronics Engineering
}

The Ground Penetrating Imaging Radar Project was established to investigate the feasibility of designing an electromagnetic (EM) radar system to examine the internal structure of concrete structures typically found in the highway industry. The central project involved the coordination of the EM modeling, imaging, code design, and experimentation efforts at Lawrence Livermore National Laboratory. The modeling effort generated data for EM imaging and enabled the precise control of individual parameters in the model.

\section{Introduction}

The modeling effort consisted of three phases: (1) complex permittivity analysis of cement using a coaxial line; (2) model construction and experimental verification in one dimension, which was represented by a coaxial line in the time domain; and (3) model construction and experimental verification in two dimensions, which was represented in the model by a slice through a concrete block and experimentally by an antenna with a fan beam.

\section{Prodress}

One-dimensional (1-D) and two-dimensional (2-D) models were constructed and compared with experimental data. The 1-D data served as a preliminary test of the dispersion algorithms added to the AMOS'1 2-1/2 D FDTD (Finite Difference Time Domain)electromagnetic (EM) modeling code. The 2-D data served as a verification for the concrete model and as a method to create waveforms for the image reconstruction algorithms.

The following parametric studies were performed:

$\begin{array}{ll}\text { Pulse width: } & 100 \mathrm{ps} \text { to } 1000 \mathrm{ps} \\ \text { Change depth: } & 10-\mathrm{mm} \text { to } 150 \text {-mm voids } \\ \text { Change size: } & 5-\mathrm{mm} \text { to } 75-\mathrm{mm} \text { voids } \\ \text { Aggregate \%: } & \begin{array}{l}10 \% \text { to } 50 \% \text { aggregate proba- } \\ \text { bility }\end{array}\end{array}$

\author{
Multi-Receiver: 5-mm to 45-mm spacing \\ (simulates highway speed or \\ prf changes) \\ Multi-Target: $\quad$ no targets, 1 void, 2 rebars, \\ 2 rebars +1 void, grate, shad- \\ owing \\ Air/Concrete: 1 case \\ Bistatic data: 1 set
}

The parametric studies gave some results that were already hypothesized 23: (1) the desired frequency is close to $2 \mathrm{GHz}$; (2) the correction filters have an image 'gain' of a factor of two; (3) large targets reradiate in addition to reflecting; (4) monostatic data spatially averages out the aggregate effects for small-and medium-sized particles; (5) bistatic data is more susceptible to aggregate effects near the transmitter than monostatic data, which is why commercial systems do not see a lot of aggregate effects; and (6) shadowing is not a significant problem as long as the spacing between the rebars is greater than three times the pulse width, and the rebars are not appreciably larger than the pulse width 4 (to give the diffracted field time to repair the wave front).

\section{1-D and 2-D Verification Effort}

A coaxial line was used for the 1-D simulation, with the cement sample embedded in a removable section of 2-in. coaxial line. The Lorentzian param- 


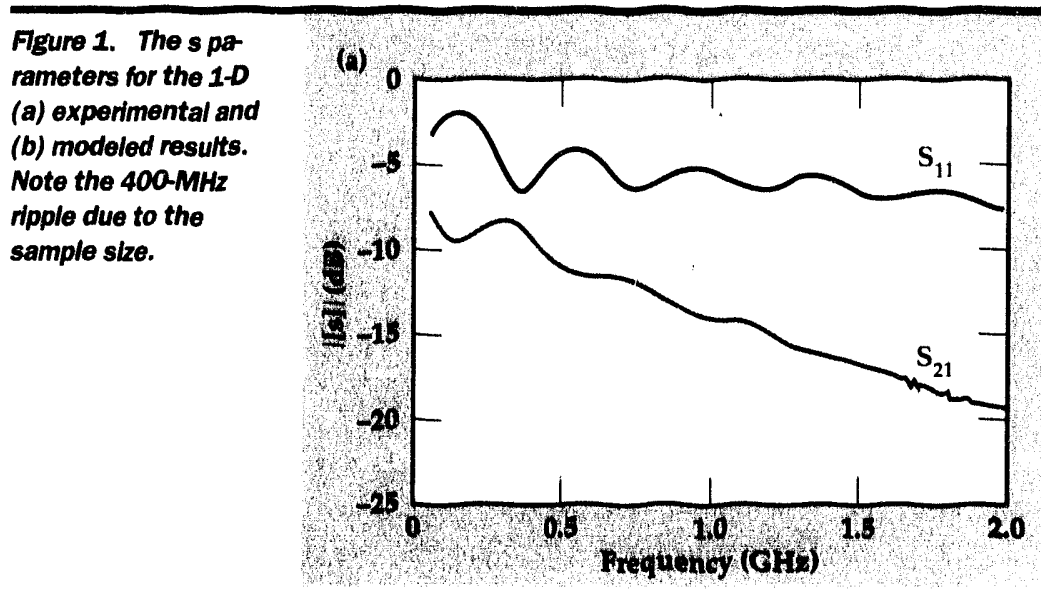

eters used for the initial 1-D and 2-D dispersion cases are as follows:

$$
\begin{aligned}
& F\left\{\sum_{i=1}^{i=\infty} \alpha_{i} e^{-\beta_{i} t} \sinh \left(\gamma_{i} t\right)\right\} \\
& =\frac{1}{2}\left[\sum_{i=1}^{t=\infty} \frac{1}{\beta_{i}-\gamma_{1}+j \omega}-\frac{1}{\beta_{i}+\gamma_{i}+j \omega}\right] \\
& \alpha_{1}=1.55 \cdot 10^{10}, \\
& \beta_{1}-\gamma_{1}=3.29 \cdot 10^{8}, \\
& \beta_{1}+\gamma_{1}=5.58 \cdot 10^{10}, \\
& \alpha_{2}=1.63 \cdot 10^{12}, \\
& \beta_{2}-\gamma_{2}=1.16 \cdot 10^{11}, \\
& \beta_{2}+\gamma_{2}=3.62 \cdot 10^{12} .
\end{aligned}
$$

The results for the 1-D experiment, performed in the Lawrence Livermore National Laboratory (LLNL) EM lab, ${ }^{5}$ are shown in Fig. 1. The 400-MHz ripple seen in Fig. 1 represents the resonance in the material sample due to its length. The

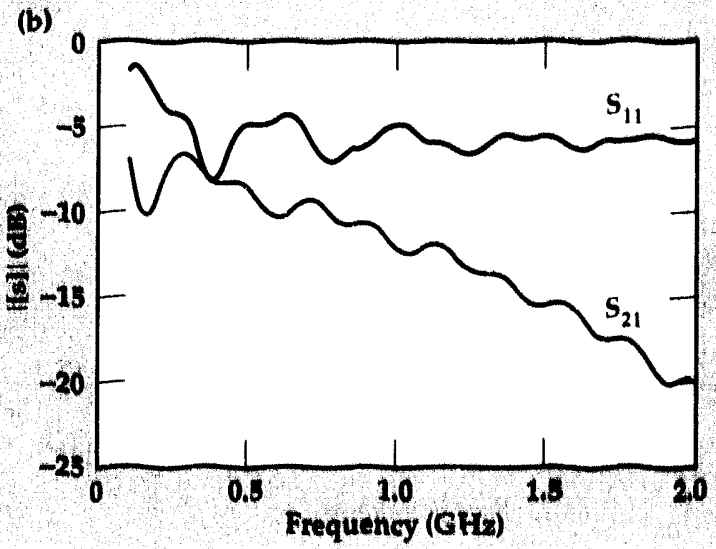

2-D verification experiment was performed in the LLNL Anechoic Chamber using a broadband antenna (with a fan beam pattern), a concrete block, a broadband field probe (Prodyne Ddot probe), and a transient digitizer. The time domain waveforms for the experimental and modeled cases are shown in Fig. 2. The antenna beam pattern, pulse shape, aggregate, and dispersion effects of the concrete block were included in the model. The finite size of the block and the diffraction around the block were also included in the model.

\section{2-D Concrete Simulation}

Figure 3 shows the received waveform from one of the receiver antennas (1 of 15), with the individual reflection identified for a geometry typical of the project. ${ }^{6}$ In this, there were two rebars and one void at different depths and cross-range distances. The effects of the aggregate in the problem are clearly visible. The aggregate is modeled as discrete scattering bodies of finitesize. The lighter

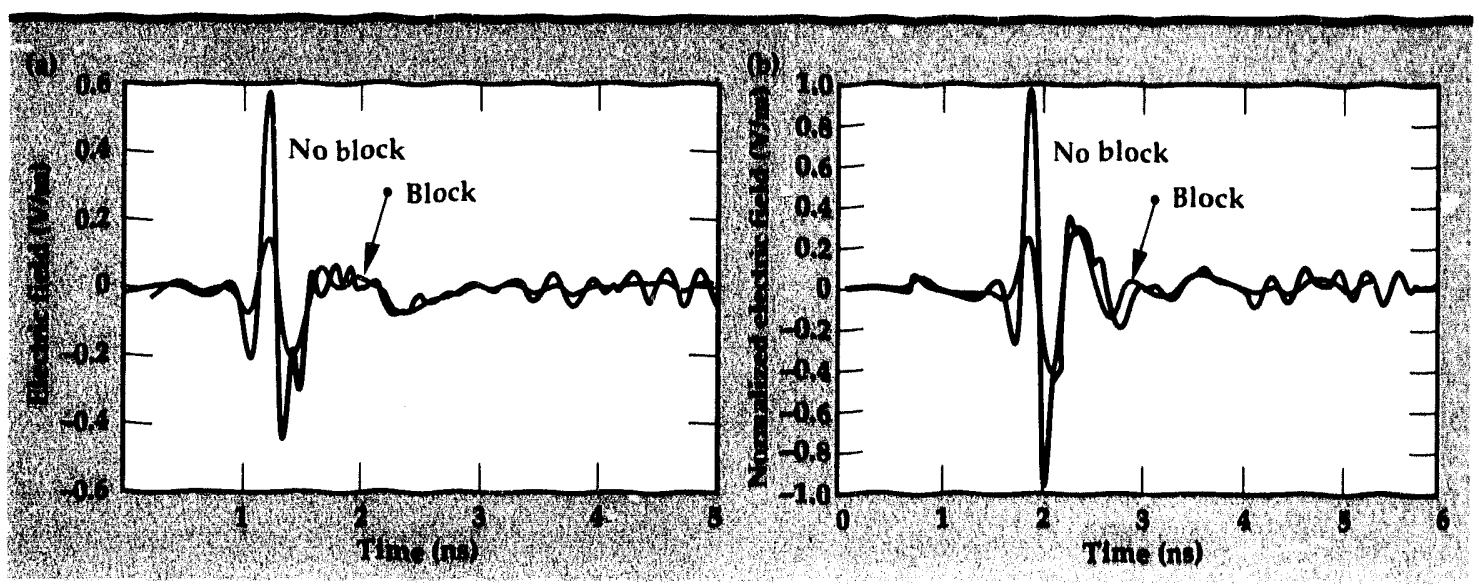

Figure 2. The time domain waveforms for the 2-D concrete block experiment compared to the modeled results. The modeled results are normalized. The negative-going double peak in the experimental results is combined into a single negativogoling peak in the modeled rosults. 


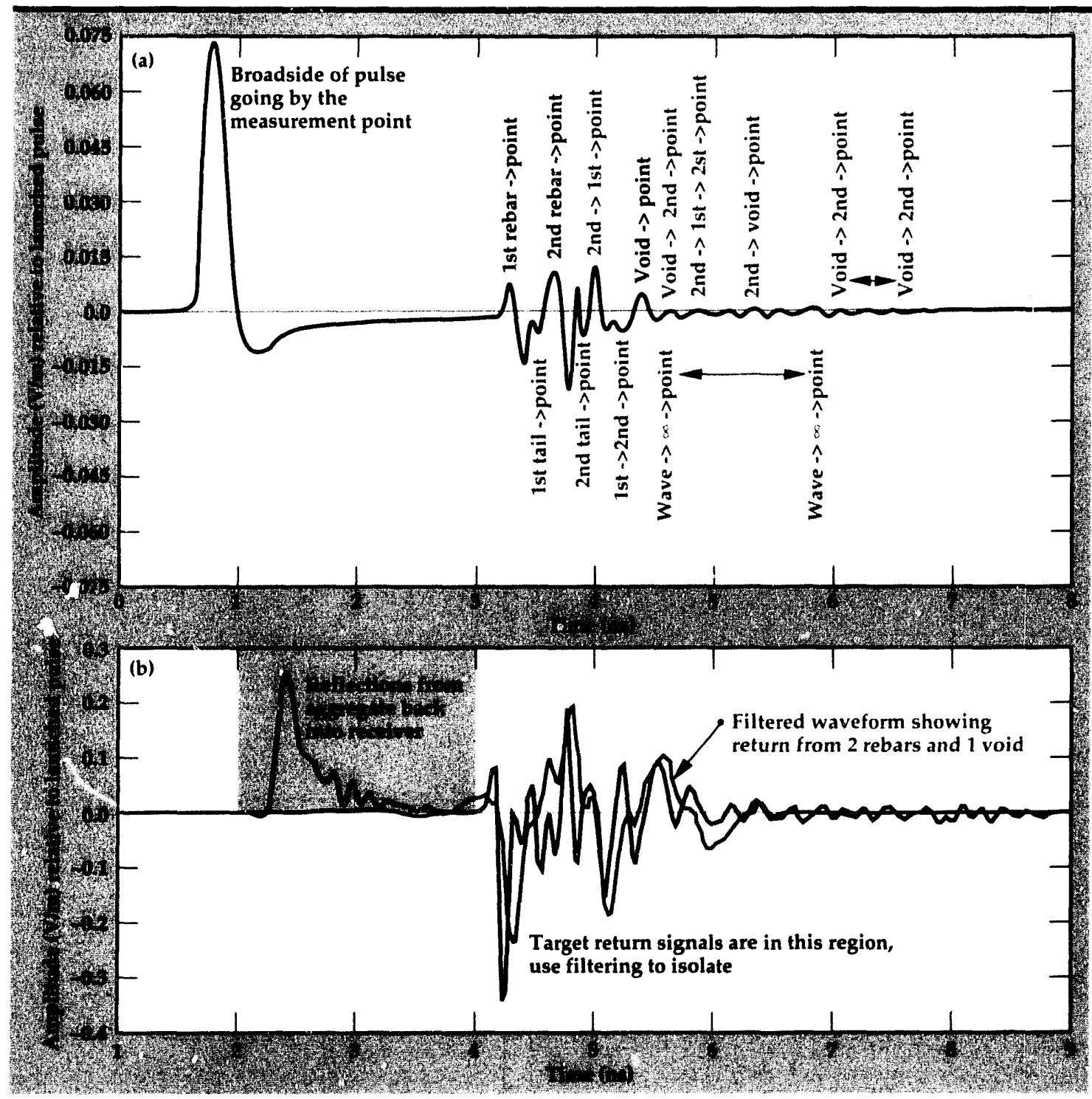

Figure 3. Recelved waveforms from one of 15 recelver antennas. The first waveform shows the recetved time domain wave form from one of the recelver elements in the modeled case with no aceregate and no dispersion. The individual target ro. flections are identifiad. The second waveform shows the effocts of the agegregate in the problom. The receiver in thits case is closer to the transmitter than in the provious case. The lighter curve shows the resulting waveform after the appllcation of the imaging team's adaptive fitter.

curve in Fig. 3 shows the results from the imaging team's adaptive filter. The reflections from the two rebars and from the void are clearly visible. The parametric study listed above was performed; Fig. 4 shows a typical wave propagation scenario. The $90^{\circ}$ beamwidth of the antenna, the two rebars, the void, and the aggregate are all visible. The aggregate radius is one half that of the rebars and represents a $30 \%$ probability duty cycle. The earlytime backward-propagating waves are due to the aggregate.

\section{Conclusions}

Aggregate sizes less than one third of the pulse width did not create significant reflections at the receivers. Aggregate sizes on the order of the pulse width created discrete waveforms in the received signals in the areas around the transmitter where the power density was the strongest. Due to the dispersion effects of the concrete, the aggregate was most reflective (in a relative sense) near the transmitter when the pulse was still short. Since 


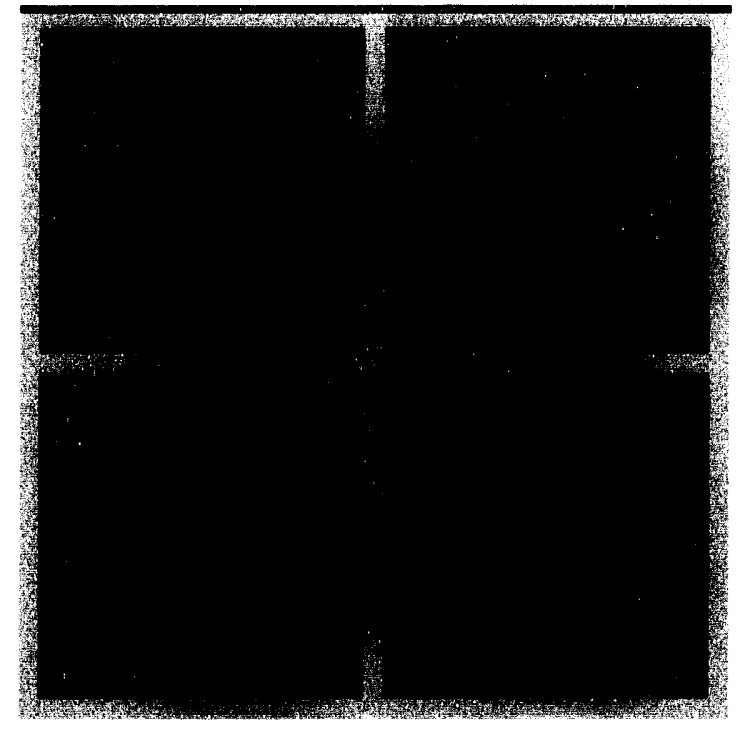

Figure 4. Four frames from a propagating EM wave se quence showing the reilections from the varlous targets. The transmit hom is on the upper surface of the concrete, and the wave propagates down into the material. The two rebars are on the rifit; the single vold is on the left. Note the polarity difierence botween the rebars and the vold. Also observe the multiple reflections between the outer targets and the center torget.

the area around the transmitter also had the greatest power density, then the maximum returned waveform (in an absolute sense) from the aggregate was also seen in this region.

This modeling effort demonstrated that the original complex permittivity data obtained in the 1-D case does support large-scale material modeling, as was expected. More important, this effort confirmed the assumptions that were made about the aggregate and its modelability. ${ }^{7}$ The size of the individual rocks constituting the aggregate and their probability distribution were more important than some exact spatial placement for each rock in the model. This result provided direct support for the usability of an adaptive filter as part of the imaging effort, to remove the aggregate effects even when individual aggregate particles generate discrete reflection waveforms in the received waveform. The aggregate specifications are known, or at least are specified, for concrete structures.

\section{Future Work}

A realistic dispersive concrete model will be introduced in three dimensions, using the TSAR code, and a realistic synthetic aperture radar (SAR) will be modeled using a section of a typical bridge deck as the target of interest. The transmitter/ receiver designs will be optimized to efficiently use the spectral information in the waveforms. Comparisons will be made to the experimental effort being performed on the $6 \mathrm{ft}-x-6 \mathrm{ft}-\mathrm{x}-1 \mathrm{ft}$ concrete test slab. This effort is already under way.

Issues that still need to be addressed are: (1) single vs multiple transmitters; (2) different antenna beamwidths based on distance from the transmitter(s); (3) a single linear array sweeping a synthetic aperture vs a real SAR; (4) optimum receiver antenna size vs receiver density; and (5) the temporal holographic use of the time domain waveforms.

\section{Acknowledgements}

Thanks go to John DeFord (LLNL) for his advice and timely modifications to the AMOS code and to Robert McLeod (LLNL) for his efforts in adding dispersion to the TSAR code. The imaging team consisted of Jose M. Hernandez (LLNL) and Joe Arellano ${ }^{8}$ (Sandia National Laboratory) with assistance from James Brase (LLNL).

1. J.T.DeFord, G. Kamin, L. Walling, and G.D.Craig, Development and Applications of Dispersive Soft Ferrite Models for Time-Domain Simulation, Lawrence Livermore National Laboratory, Livermore, California, UCRL-JC-109495 (1992).

2. K. Olp, G. Otto, W.C.Chew, and J.F. Young, J. Mater. Sci. 26, 2978 (1991).

3. K.S. Cole and R.H. Cole, I. Chem. Phys., 341 (April 1941).

4. M. Kanda, IEEE Trans. Antennas Propag., 26, 439.

5. C. Avalle, Broadband Complex Permittivity Measurements of Cement, Lawrence Livermore National Laboratory, Livermore, California (in preparation).

6. R. Zoughi, G.L. Cone, and P.S. Nowak, "Microwave Nondestructive Detection of Rebars in ConcreteSlabs," Materials Evaluation-American Society for Nondestructive Testing, 1385 (November 1991).

7. K.R. Maser, "Detection of Progressive Deterioration in Bridge Decks Using Ground Penetrating Radar," Proc. ASCE Convention (Boston, Massachusetts), (October 27, 1986).

8. J. Arellano, "Adaptive Filter for LLNL's Impulse Radar Inspection of Roadways and Bridges," EE373A, Adaptive Signal Processing (Winter 199192). 


\title{
Band Gap Engineering for Infrared Detectors
}

\author{
J. Brian Grant \\ Engineering Research Division \\ Electronics Engineering
}

We have extended and improved modeling codes for strained layer superlattices. Significant improvements include capabilities for reliable subband tracing and multilayer modeling; better validation of eigenstates; and the calculation of physical quantities such as wave function and opticai absorption profiles and effective masses.

\section{Introdiction}

Applications for infrared (IR) detectors include military, civilian, and medical devices with current interest focused on the far IR spectrum (wavelengths greater than $10 \mu \mathrm{m}$ ). Small band gaps corresponding to this range push the engineering parameters in commercial IR detector technology, which is alloying $\mathrm{Hg}_{1-\mathrm{x}} \mathrm{Cd}_{\mathrm{x}} \mathrm{Te}$. This process is not only very sensitive to composition, but toxic and volatile too. While a switch from alloy to superlattice technology would relax the conditions on exact composition, 1 an even greater benefit is obtained from a switch to III-V semiconductors, ${ }^{2}$ where internal strain can be used in designing band gaps. Recent advances in $\mathrm{Ge}_{1-x} \mathrm{Sb}_{\mathrm{x}}$ alloy fabrication provide another alternative ${ }^{3}$ which is less sensitive to alloy composition. Severe lattice mismatches prevent the formation of $\mathrm{Ge} / \mathrm{Sb}$ superlattices.

Of particular interest are superlattices of $\mathrm{GaSb} /$ $\mathrm{In}_{1-\mathrm{x}} \mathrm{Al}_{\mathrm{x}} \mathrm{As}$ in which the alloy composition controls lattice mismatch, the source of internal strain. The major tradeoff is reduced optical absorption because the superlattice is type II, i.e., the conduction and valence states are principally confined to different material layers. Thinner layering and strain help to overcome this by increasing tunneling and consequently increasing wave function overlaps.

The modeling of strained layer superlattices is perhaps best achieved through the use of interface transfer matrices. These matrices identify how wave functions in one bulk-like material are transformed into those of the next. While this idealizes interfaces, it eliminates the excessive basis size of traditional approaches, which must model each atom in the periodic structure. The matrix approach also allows modeling of non-periodic, finite-sized structures.

Computational speed is further enhanced by use of the k.p theory, which expands bulk wave functions in terms of those at the Brillouin zone center. Because interest is in the lowest conduction and highest valence, i.e, in bands near the Brillouin zone center, only the eight spin split s- and p-wave functions of the bulk are kept. Löwden perturbation theory is used to extend the range of accuracy by including effects of other wave functions to first-order.

\section{Progress}

A significant portion of our project has focused on extending and improving existing modeling codes., 5 By combining several codes into a single package and by reducing and simplifying required

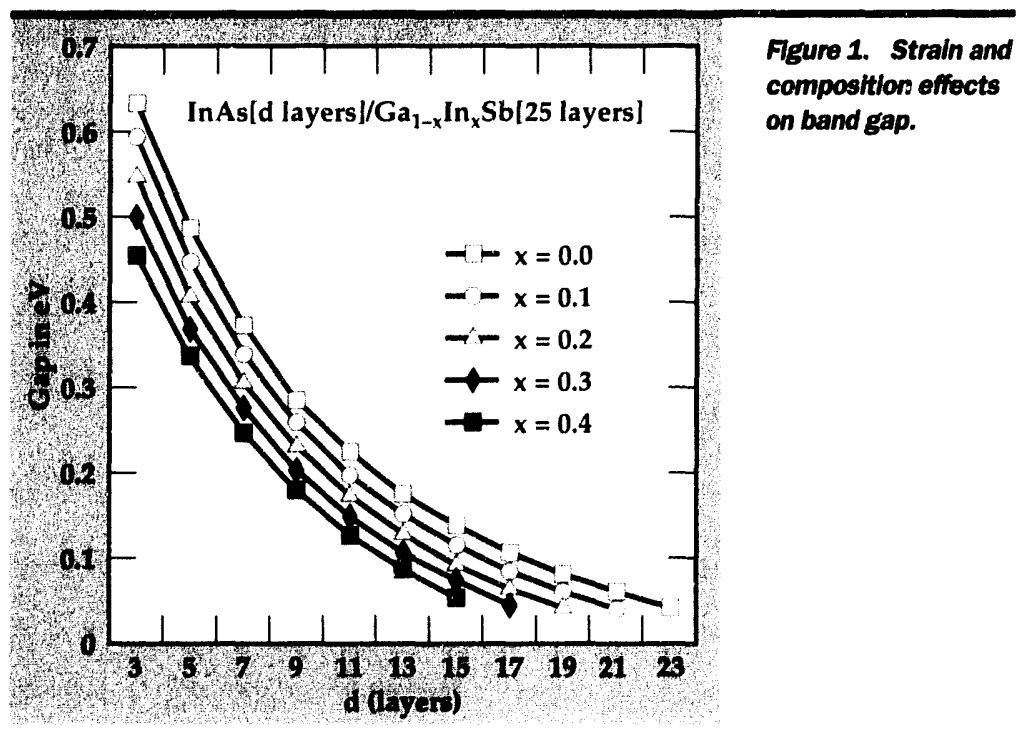




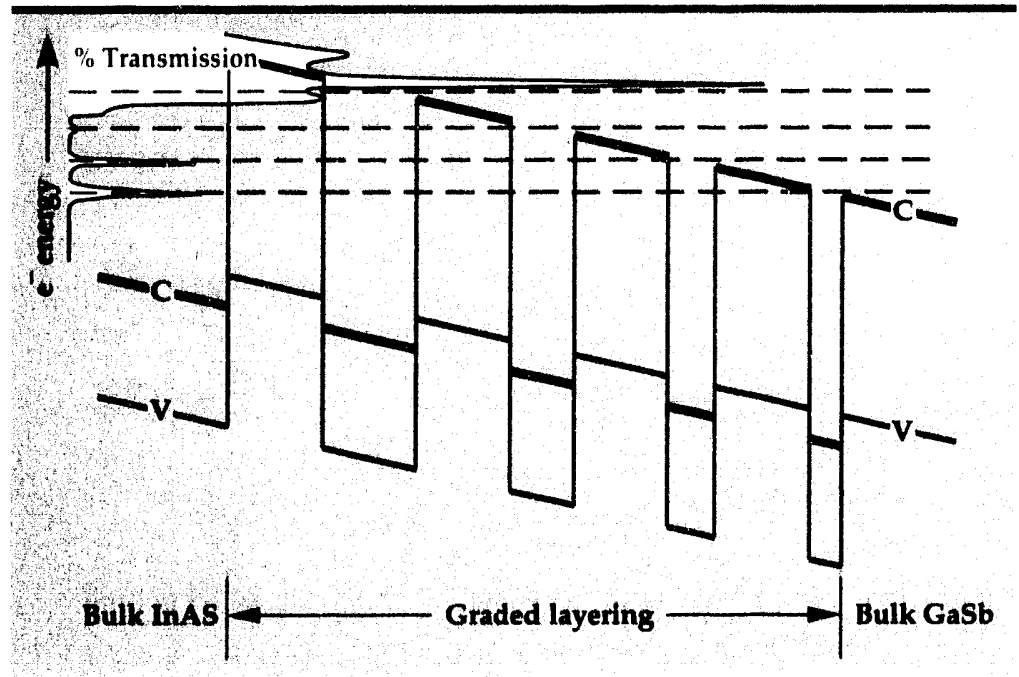

Figure 2. Percent transmission as a function of electron energy relative to the bulk conduction (C) and valence (V) band edges. Slope of band edges Indicates an applled voltage.
Figure 1 shows a sample calculation for SBRC that demonstrates the effects of strain (alloy) and layer thickness on band gaps in InAs / $\mathrm{Ga}_{1-1} \ln , \mathrm{Sb}$ superlattices. Note that a target wavelength of $12 \mu \mathrm{m}$ would require very thick layers of InAs without alloying $(x=0.0)$, but that only 11 or 12 atomic layers would be required for a composition where $x=0.4$. In addition to the band gap, SBRC is interested in the actual position of the conduction band with respect to the chemical potential (Fermi level) and the optical absorption as functions of the same parameters. Similar plots can be made of those values. Values of effective masses calculated by the sls code can also be used for additional calculations by SBRC.

Modeling for programs at Lawrence Livermore National Laboratory (LLNL) has been based on graded-layer superlattices that slowly accommodate large band offsets. Externally applied voltages then provide a rather constantenergy conduction band throughout the structure. The sls code easily identifies acceptabletransmission energy ranges, as shown in Fig. 2, as well as resonant tunneling states, which build up much larger electron concentrations between barrier layers. While this code is unable to provide self-consistent estimates of current densities, the information available can provide a guide to superlattice grading, and quantitative estimates of the necessary external voltages.

\section{Future Work}

This project has included software development and improvement, modeling, and technology transfer. The result is a rather complete and usable strained-layer superlattice code. Relations with SBRC are continuing, supported in part by the Physics Department at LLNL.

1. D.L. Smith, T.C. McGill, and J.N. Schulman, Appl. Plyys. Lett. 43, 180 (1983).

2. D.K. Arch, G. Wicks, T. Tonac, and J.-L. Staudenmann, I. Appl. Plyys. 58, 39333(1985).

3. S.M. Lee and W. Paul, "Electronic Band Gap Measurements of Bulk Metastable Crystalline $C_{e}$, Sn, Alloys, $(0 \leq x \leq 0.311$," preprint.

4. C. Mailhiot and D.L. Smith, Crit. Re's, Solit State' Mater. Sci. 16, 131 (1990)).

5. D.L. Smith and C. Mailhiot, Rez. of Mot. Mhyss. 62 , $173(1990)$. 

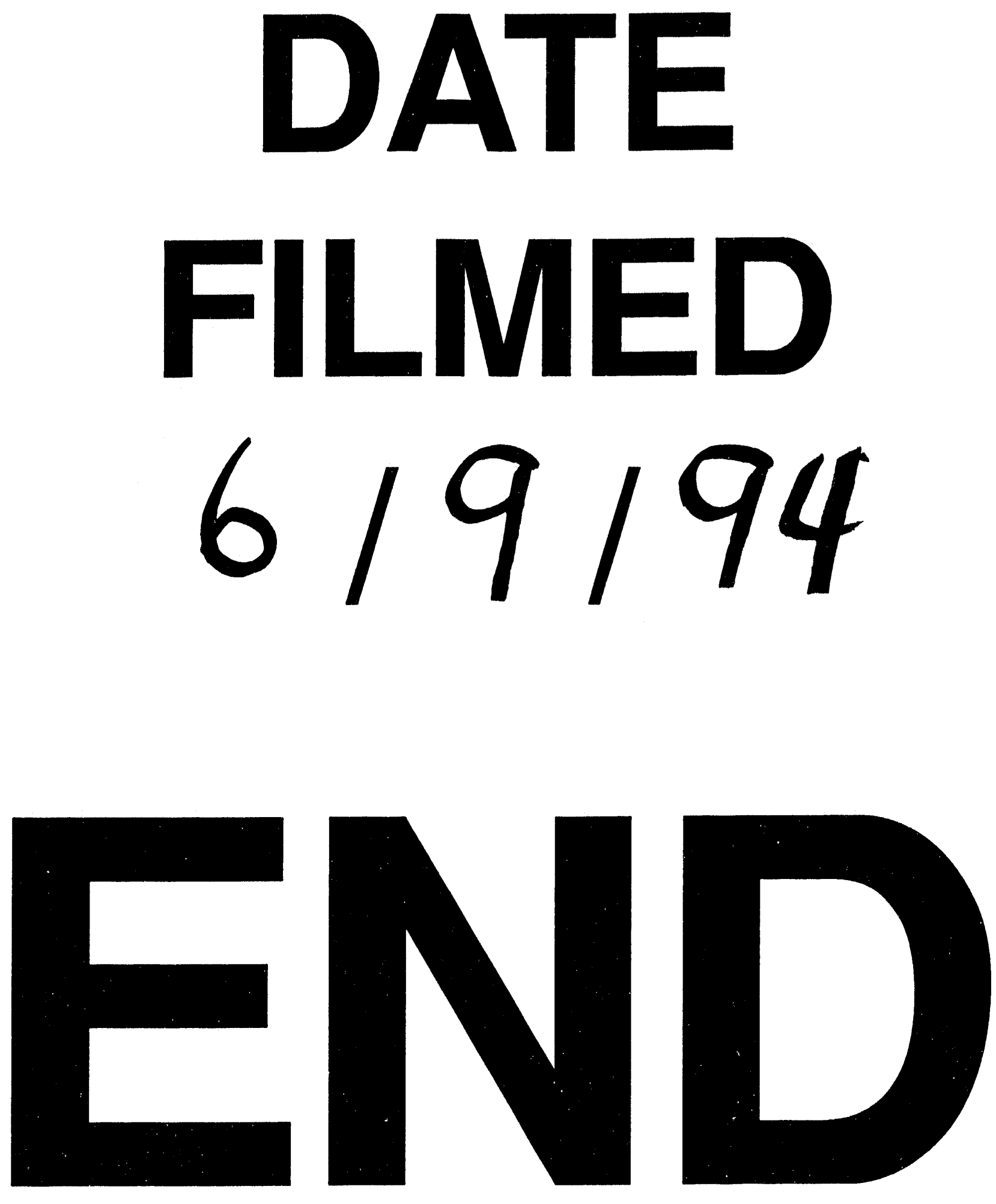
